\title{
THE INFLUENCE OF ALIPHATICS ON SOOT INCEPTION
}

\section{MODELLING}

\author{
By \\ Nemanja Ceranic, B. Eng \\ Mechanical Engineering \\ Ryerson University, 2018 \\ A thesis presented to Ryerson University \\ in partial fulfillment of the \\ requirements for the degree of \\ Master of Applied Science \\ in the program of \\ Mechanical Engineering
}

Toronto, Ontario, Canada

(C) Nemanja Ceranic 2018 


\section{Author's Declaration}

I hereby declare that I am the sole author of this thesis. This is a true copy of the thesis including any required final revisions, as accepted by my examiners.

I authorize Ryerson University to lend this thesis to other institutions or individuals for the purpose of scholarly research.

I further authorize Ryerson University to reproduce this thesis by photocopying or by other means, in total or in part, at the request of other institutions or individuals for the purpose of scholarly research.

I understand my thesis may be made electronically available to the public. 


\title{
Abstract \\ The Influence of Aliphatics on Soot Inception Modelling
}

\author{
Master of Applied Science, 2018 \\ Nemanja Ceranic \\ Mechanical Engineering \\ Ryerson University
}

Soot models have been investigated for several decades and many fundamental models exist that prescribe soot formation in agreement with experiments and theories. However, due to the complex nature of soot formation, not all pathways have been fully characterized. This work has numerically studied the influence that aliphatic based inception models have on soot formation for coflow laminar diffusion flames. CoFlame is the in-house parallelized FORTRAN code that was used to conduct this research. It solves the combustion fluid dynamic conservation equations for a variety of coflow laminar diffusion flames. New soot inception models have been developed for specific aliphatics in conjunction with polycyclic aromatic hydrocarbon based inception. The purpose of these models was not to be completely fundamental in nature, but more so a proofof-concept in that an aliphatic based mechanism could account for soot formation deficiencies that exist with just PAH based inception. The aliphatic based inception models show potential to enhance predicative capability by increasing the prediction of the soot volume fraction along the centerline without degrading the prediction along the pathline of maximum soot. Additionally, the surface reactivity that was used to achieve these results lied closer in the range of numerically derived optimal values as compared to the surface reactivity that was needed to match peak soot concentrations without the aliphatic based inception models. 


\section{Acknowledgements}

I would like to express my appreciation for my supervisor Dr. Seth B. Dworkin. His dedication and passion were a source of inspiration coupled with invaluable guidance made this research possible and enjoyable throughout.

I would like to thank the Dworkin research group and my fellow graduate students that have provided countless hours of entertainment and whom with I have shared many experiences during my time here at Ryerson University.

I would like to convey my gratitude towards my parents for their moral and financial support that have made this academic endeavour possible.

I would like to acknowledge SciNet HPC Consortium for providing computational resources. SciNet is funded by: the Canada Foundation for Innovation under the auspices of Compute Canada; the Government of Ontario; Ontario Research Fund - Research Excellence; and the University of Toronto. I also acknowledge the Natural Sciences and Engineering Research Council of Canada, Province of Ontario, and Ryerson Faculty of Engineering and Architectural Science for financial support. 


\section{Table of Contents}

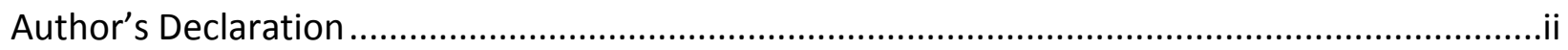

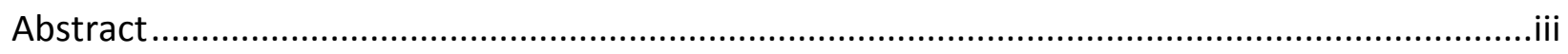

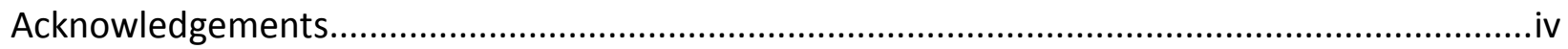

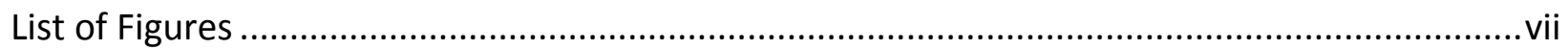

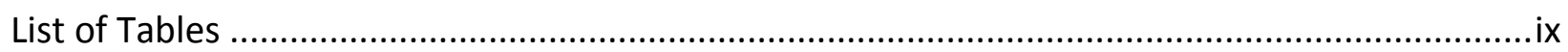

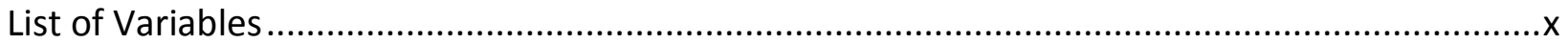

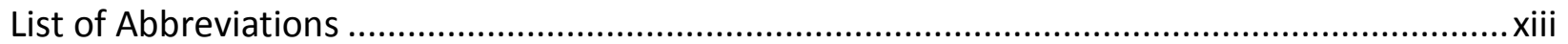

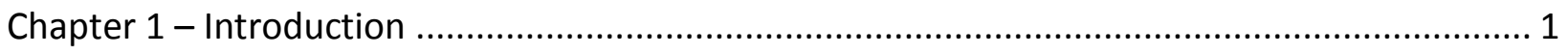

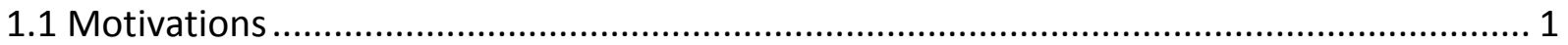

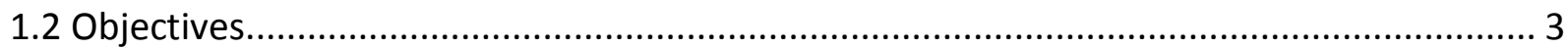

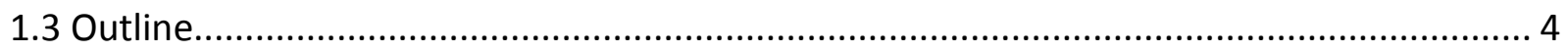

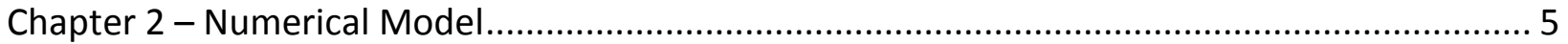

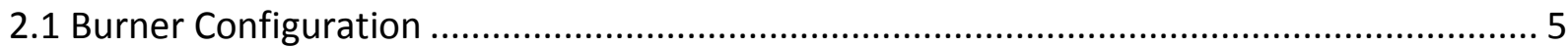

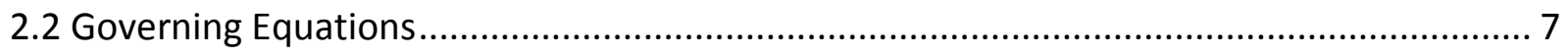

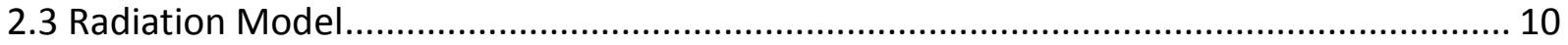

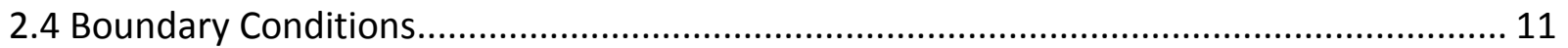

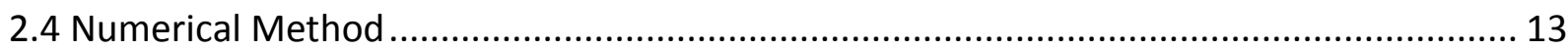

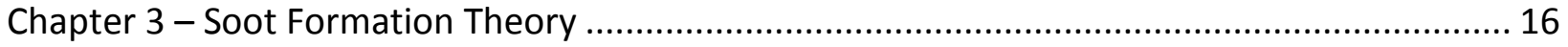

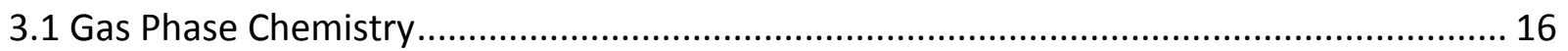

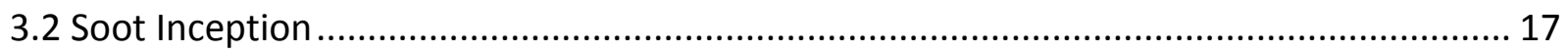

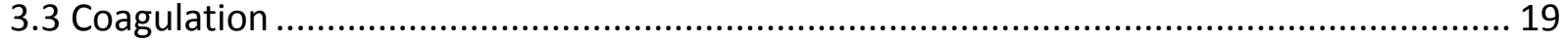

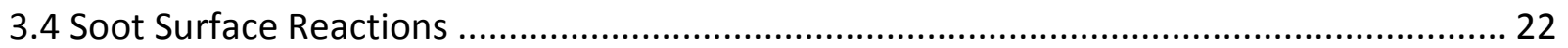

3.4.1 Hydrogen-Abstraction-Carbon Addition and Oxidation ....................................... 22

3.4.2 Polycyclic Aromatic Hydrocarbon Condensation................................................. 24

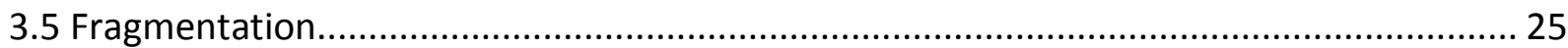

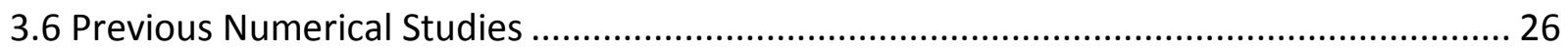

3.6.1 Influence of the Chemical Kinetic Mechanism ................................................... 26

3.6.2 Application to Partially Premixed Systems ............................................................ 29

3.6.3 Application to Different Fuel Carbon Composition ............................................... 33

Chapter 4 - Aliphatic Model Development, Testing, and Results .......................................... 35 


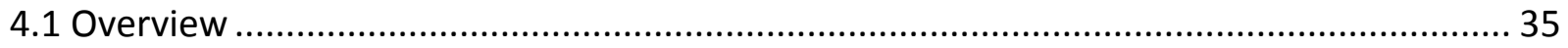

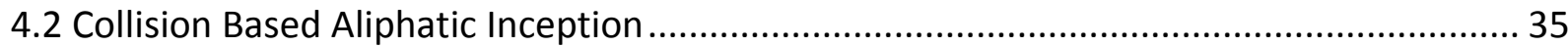

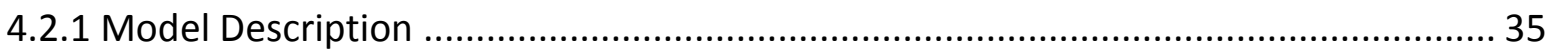

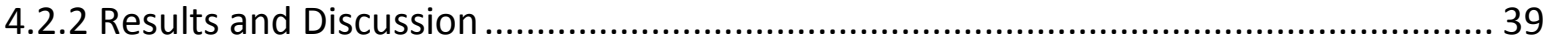

4.3 Collision Based Aliphatic Stabilization ....................................................................... 46

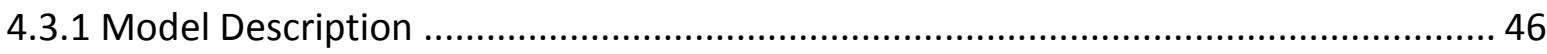

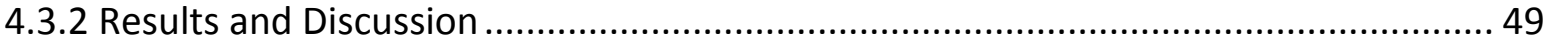

4.4 Model Comparison with Experimental Results .......................................................... 59

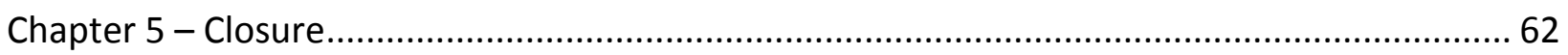

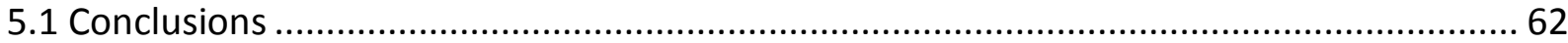

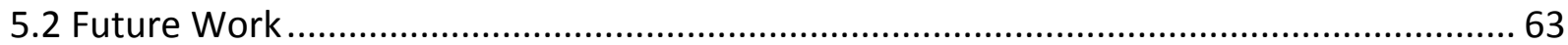

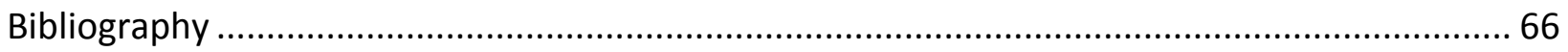




\section{List of Figures}

Figure 1: Predicted soot volume fraction as a function of height above the burner for the Appel et al. [13] and Dworkin et al. [11] (present mechanism) mechanisms along the centerline of the Santoro Non-Smoking II flame. Experimental results are shown from [15]. Reproduced from [11] 3

Figure 2: Coflow burner schematic. 5

Figure 3: Coflow burner schematic with boundary conditions present. 11

Figure 4: Staggered mesh where the velocity components are stored the cell faces of the pressure control volumes.

Figure 5: Predicted soot volume fraction as a function of height above the burner along the centerline for the Slavinskaya and Frank [45] mechanism, Appel et al. mechanism [13], and experimental data along the centerline of the NSII flame [24]. Reproduced from Dworkin et al. [11].

Figure 6: Relative mass contribution for the McEnally and Pfefferle [12] ethylene partially premixed flame along the centerline for different equivalence ratios. Reproduced from Chernov et al. [10]. 30

Figure 7: Relative value for McEnally and Pfefferle [60] ethylene partially premixed flame along the centerline for different equivalence ratios. Reproduced from Chernov et al. [10]. 31 Figure 8: Relative mass contribution for Arana et al. [59] ethylene partially premixed flame along the wings for different equivalence ratios. Reproduced from Chernov et al. [10]. 32 Figure 9: Visual representation of the collision based aliphatic inception model moving two aliphatic species, which in this case would be $\mathrm{C}_{4} \mathrm{H}_{6}$, from the gas phase to the solid soot phase.

Figure 10 Predicted soot volume fraction as a function of height above the burner for a collision efficiency of $1 \mathrm{e}-11$ with constant surface reactivity of 0.3 along the wings of the NSII flame. The results of a model without aliphatic inception and a surface reactivity of 0.85 is shown alongside experimental results from [15]....

Figure 11: Predicted soot volume fraction as a function of height above the burner for a collision efficiency of $1 \mathrm{e}-11$ with constant surface reactivity of 0.3 along the centerline of the NSII flame. The results of a model without aliphatic inception and a surface reactivity of 0.85 is shown alongside experimental results from [15]

Figure 12: Predicted soot volume fraction as a function of height above the burner for a model without aliphatic inception using $\alpha$ of 0.85 and 1.0 along the wings of the methane flame. The aliphatic inception model is shown using the same $\beta$ as for the NSII, 1e-11. The experimental work of [29] is plotted.

Figure 13: Predicted soot volume fraction as a function of height above the burner for a model without aliphatic inception using $\alpha$ of 0.85 and 1.0 along the centerline of the methane flame. The aliphatic inception model is shown using the same $\beta$ as for the NSII, 1e-11. The experimental work of [29] is plotted. 
Figure 14: Visual representation of the collision based aliphatic stabilization model moving two aliphatic species, which in this case would be $\mathrm{C}_{4} \mathrm{H}_{6}$, from the gas phase to the solid soot phase.

Figure 15: Computed aliphatic species concentrations using the original model and a surface reactivity of 0.85 for the NSII flame. The superimposed aliphatic field represents the summation of the aliphatic species presented. The results are expressed in ppm.

Figure 16: Computed PAH species concentrations using the original mechanism and a surface reactivity of 0.85 for the NSII flame. The results are expressed in ppm. Additionally, the computed collision efficiency of the form $f(x 1, x 2, x 3)=0.1139[x 1+x 2+x 3]$ is plotted.

Figure 17: Predicted soot volume fraction as a function of height above the burner for the stabilization model with a collision efficiency prescribed by $f\left(x_{1}, x_{2}, x_{3}\right)=0.1139\left[x_{1}+x_{2}+x_{3}\right]$ along the wings and centerline of the NSII flame. The results of the original model are shown alongside experimental results from [15]. The surface reactivity was constant between the two models and had a value of 0.85 . The collision efficiency for the original model was a constant value of $1 \mathrm{e}-4$.

Figure 18: Predicted soot volume fraction as a function of height above the burner for the model with a collision efficiency prescribed by $f(x 1, x 2, x 3)=0.002278[x 1+x 2+x 3]$ along the wings and centerline of the NSII flame. The results of the original model without aliphatic inception are shown alongside experimental results from [15]. The surface reactivity was constant between the two models and had a value of 0.85 . The collision efficiency for the original model was a constant value of $1 \mathrm{e}-4$.

Figure 19: Predicted soot volume fraction as a function of height above the burner for the model with a collision efficiency prescribed by $f(x 1, x 2, x 3)=0.059228[x 1+x 2+x 3]$ along the wings and centerline of the NSII flame. The results of the original model without aliphatic inception are shown alongside experimental results from [15]. The surface reactivity for the original model was 0.85 whereas the stabilization model used a surface reactivity of 0.3 . The collision efficiency for the original model was a constant value of $1 \mathrm{e}-4$.

Figure 20: Predicted soot volume fraction as a function of height above the burner for the best results of the stabilization model, inception model, original model, and experimental results from [15].

Figure 21: Predicted soot volume fraction as a function of height above the burner for the best results of the stabilization model, inception model, original model, and experimental results from [15]. 


\section{List of Tables}

Table 1: Santoro et al. $[15,24]$ Flow Conditions........................................................................... 6

Table 2: HACA and Oxidation Reaction Sequence ................................................................. 23

Table 3: Collision Based Aliphatic Inception Iterations Results for the NSII Flame ..................... 40

Table 4: Magnitude Factor Iteration Scheme of the Linear Function for the NSII flame ............ 55

Table 5: Correction Factor Iteration Scheme of the Linear Function for the NSII flame............. 56

Table 6: Iteration Scheme of the Linear Function for NSII Flame with an $\alpha=0.3 \ldots \ldots \ldots \ldots \ldots \ldots . . . . . . .58$ 


\section{List of Variables}

Latin

A - Coefficient that governs the overall fragmentation rate

$A_{v}-$ Avogadro's number

$\mathrm{C}_{\mathrm{c}}(\mathrm{Kn})$ - Cunningham slip correction factor

$\mathrm{Cg}_{\mathrm{g}}$ - Concentration of colliding gaseous species $\left(\mathrm{C}_{2} \mathrm{H}_{2}\right)$

$c_{p}$ - Mixture specific heat capacity at constant pressure

$c_{p, k}-$ Specific heat capacity of the $k^{\text {th }}$ species

$c_{p, s}-$ Specific heat capacity of soot

D - Diffusion coefficient

$D_{f}-$ Fractal dimension

$D_{i}^{a}-$ Particle diffusion

$D_{j}$ and $D_{k}$ - Diffusion coefficients for soot particles in the $j^{\text {th }}$ and $k^{\text {th }}$ sections

$d_{m}-$ Mobility diameter

$f$-Volume filling factor

$f_{D}-$ Transition regime correction factor

$g_{z}-$ Gravitational acceleration

$h_{k}-$ Specific enthalpy of the $k^{\text {th }}$ species

$h_{s}$ - Specific enthalpy of soot

I - Spectral intensity

$\mathrm{I}_{\mathrm{B}}$ - Spectral black-body intensity

$\mathrm{I}_{g, \mathrm{i}}$ - Total surface growth rate of PAH condensation and HACA for the $\mathrm{i}^{\text {th }}$ section

$\mathrm{I}_{\mathrm{ox}, \mathrm{i}}$ - Oxidation rate for the $\mathrm{i}^{\text {th }}$ section

$\mathrm{k}_{\mathrm{B}}-$ Boltzmann constant

$\mathrm{kg}_{\mathrm{g}, \mathrm{s}}-$ Per-site rate coefficient

1-Azimuthal angle index

$\mathrm{m}$ - Polar angle index 
$m_{i}$ - Representative mass of the $i^{\text {th }}$ section aggregate

$\mathrm{N}_{\mathrm{i}}^{\mathrm{a}}$ - Aggerate number density

$\mathrm{Ni}^{\mathrm{p}}$ - Soot primary particle number density

$\mathrm{N}_{\mathrm{i}}$ - Number density of the $\mathrm{i}^{\text {th }}$ particle

$n_{p}-$ Number of primary particles in the aggregate

$\mathrm{p}$-Pressure

$\mathrm{Q}_{\mathrm{r}}$ - Radiative heat transfer by soot, $\mathrm{H}_{2} \mathrm{O}, \mathrm{CO}_{2}$, and $\mathrm{CO}$

r-Radial position

$r_{A}$ and $r_{B}-$ Radii of the two-colliding species

$\mathrm{R}_{\mathrm{abs}}-$ Absorbing sphere cluster radius

$\mathrm{R}_{\mathrm{f}}$ - Outer radius of an aggregate

$r_{o x, s}-$ Rate of removal of soot mass per unit soot surface area

$r_{p}$ - Primary particle radius

$\mathrm{S}_{\mathrm{i}}$ - Surface of the $\mathrm{i}^{\text {th }}$ particle

$\mathrm{S}_{\mathrm{i}}$ - Fragmentation rate of the $\mathrm{i}^{\mathrm{th}}$ section

T- Temperature

$u-$ Axial velocity

$v-$ Radial velocity

$\mathrm{V}_{\mathrm{s}, \mathrm{r}}-$ Soot radial diffusion velocity

$\mathrm{V}_{\mathrm{s}, \mathrm{z}}$ - Soot axial diffusion velocity

$\mathrm{V}_{\mathrm{Ts}, \mathrm{r}}$ - Radial thermophoretic velocity

$\mathrm{V}_{\mathrm{Ts}, \mathrm{z}}-$ Axial thermophoretic velocity

$W_{k}-$ Molecular weight of the $k^{\text {th }}$ species

$W_{s}$ - Molecular weight of soot

$x_{i}$ - Concentration of the $i^{\text {th }}$ species

$Y_{k}-$ Mass fraction of the $k^{\text {th }}$ species

$\mathrm{Y}_{\mathrm{s}}$ - Mass fraction of soot 
$z-$ Axial position

\section{Greek}

$\alpha$ - Surface reactivity

$\alpha-$ Geometric constant

$\beta-$ Collision efficiency

$\beta_{\mathrm{j}, \mathrm{k}}-$ Collision kernel of two aggregates in the $\mathrm{j}^{\text {th }}$ and $\mathrm{k}^{\text {th }}$ sections

$\Gamma_{i, i}$ and $\Gamma_{i, i+1}-$ Breakage distribution functions

$\delta-$ Kronecker delta function

$\eta_{p, i}-$ Number of primary particles per aggregate in the $i^{\text {th }}$ section

$\mathrm{K}$ - Spectral absorption coefficient

$\lambda$ - Mixture thermal conductivity

$\lambda_{m f p}-$ Mean free path of the gas

$\mu$ - Dynamic viscosity

$\mu$ and $\xi-$ Directional cosines

$\mu_{A B}-$ Collisional reduced mass for the two-colliding species

$\xi_{j, k}$ - Coagulation efficiency of two aggregates in the $\mathrm{j}^{\mathrm{th}}$ and $\mathrm{k}^{\text {th }}$ sections

$\rho$ - Mixture density

$\phi-$ Primary equivalence ratio

$\chi_{s}-$ Number density of surface sites

$\omega-$ Weighting function

$\omega_{k}$ - Production rate of the $k^{\text {th }}$ species

\section{Subscripts}

co - PAH condensation

$\mathrm{fr}$ - Fragmentation

nu - Nucleation

ox - Oxidation

sg - Surface growth 


\section{List of Abbreviations}

BAPYR - Benzo(a)pyrene

BAPYRS - Secondary benzo(a)pyrenyl

BGHIF - Benzo(ghi)fluoranthene

HACA - Hydrogen-Abstraction-Carbon-Addition

HPC - High performance computing

NSII - Non-Smoking II

PAH - Polycyclic Aromatic Hydrocarbon 


\section{Chapter 1 - Introduction}

\subsection{Motivations}

Any form of incomplete hydrocarbon combustion leads to the generation of soot. These particles are hazardous to human health [1, 2] as well as dangerous for the environment $[3,4,5]$. Thus, it is necessary that the characterization of soot formation be well understood, to be able to better design more environmentally friendly combustion devices. One of the advantages that numerical modelling can provide is that it allows researchers to test new theories at virtually no cost compared to experimental techniques. However, numerical combustion models require extensive knowledge of soot formation to obtain meaningful results.

Soot formation is a complex process consisting of polycyclic aromatic hydrocarbon (PAH) gas-phase growth, particle nucleation, surface growth via surface reaction, $\mathrm{PAH}$ condensation, surface oxidation, particle coalescence, particle coagulation, and fragmentation [6]. The formation and growth pathways mentioned are widely considered by the scientific community to be the accepted routes of soot generation, however, due to its complex nature, other pathways may exist that have yet to be discovered.

Soot models have been the subject of discussion for several decades and continue to improve to this day. The three types of numerical models are empirical, semi-empirical, and predominantly fundamental models. Semi-empirical soot models $[7,8]$ may have a foundation in the physics behind soot formation but lack the fundamental physics as they rely on tunable parameters. Although these models may not accurately portray what is occurring inside of the flame, they do provide scientific insights. They are also an essential milestone towards the creation of a fully fundamental model. 
One such model is the Hydrogen-Abstraction-Carbon-Addition (HACA) growth mechanism introduced by Frenklach and Wang [9]. This model traditionally uses surface reactivity ( $\alpha$ ) as a tunable constant or function for matching numerical peak soot predictions to experimental peaks [9]. Therein lies the issue that current soot models face, which is, although peak soot may be correctly predicted, other regions of the flame may not be correctly predicted through the same tuning of $\alpha$. It has been postulated that the centerline of coflow laminar diffusion flames are dominated by inception whereas the wings are dominated by surface growth through the HACA mechanism [10]. In the literature, the wings refer to the pathline of maximum soot, which tends to occur relatively close to the flame front. By tuning $\alpha$ to experimental peaks, which occur along the wings, the prediction in that region may be correct. However, many models do not have sufficient reaction chemistry or appropriate sub-mechanisms to accurately capture the trends along the centerline, and tend to under predict soot formation in that region $[11,12,13,14]$. Figure 1 shows that while comparisons of predicted and experimental soot concentration along the centerline of diffusion flames has improved via chemical mechanism development, in some cases they are still off by a factor of ten, and there may still be physical phenomena that are unaccounted for in the modelling literature. 


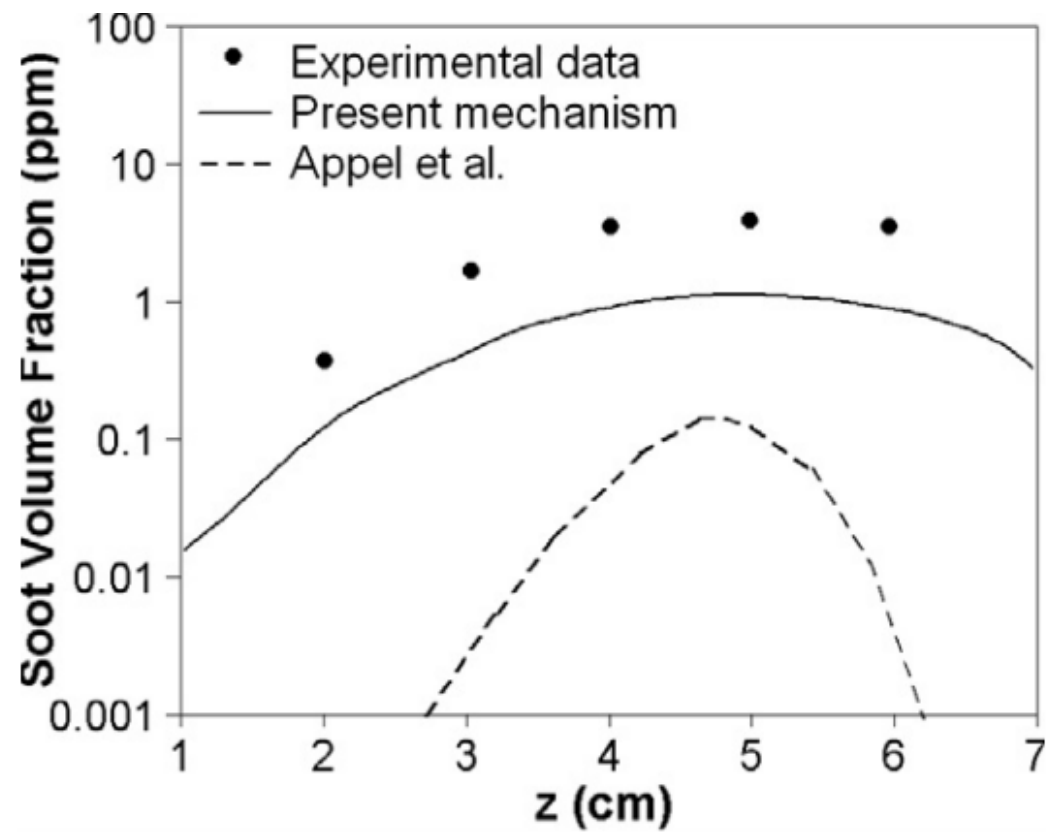

Figure 1: Predicted soot volume fraction as a function of height above the burner for the Appel et al. [13] and Dworkin et al. [11] (present mechanism) mechanisms along the centerline of the Santoro Non-Smoking II flame. Experimental results are shown from [15]. Reproduced from [11].

\subsection{Objectives}

Aliphatics, which are noncyclic hydrocarbons, have been investigated in the literature both experimentally and numerically to examine their influence on soot formation. Recent literature suggests that soot can undergo mass growth in the absence of gas phase hydrogen atoms and nascent soot can be rich in aliphatic molecules in premixed flames $[16,17,18,19]$. Furthermore, studies $[20,21,22]$ have shown that PAHs with aliphatic chains of 3 carbons and higher are more likely to result in the formation of soot compared to the same PAHs without those chains present. Aliphatics do play a role in the formation of soot, however, due to the complex nature of combustion, all the roles they play may have yet to be discovered.

The objective of this work is to investigate the influence that aliphatics may have on soot formation through specific routes. It is proposed that collision based aliphatic inception and collision based aliphatic stabilization may have some influence on soot mass growth. Two semiempirical models have been generated to investigate the impacts that each has on soot 
formation for a variety of coflow laminar diffusion flames. More details on the physics and assumptions of the models will be explained in the following sections. The models are applied to both ethylene/air and methane/air coflow laminar diffusion flames that use the same burner dimensions. It was of interest to investigate different carbon fuels to observe the effects that changes to the numerical model would have on the results for different fuels. Each fuel decomposes differently when undergoing combustion, and changes to the numerical model may not significantly impact the results of one fuel but may have significant impacts on another. Two parameters are key to these models which are the surface reactivity and the collision efficiency (B).

\subsection{Outline}

Chapter 2 provides a description of the numerical model with regards to the computational fluid dynamic aspects. Chapter 3 will discuss the necessary background and literature of soot formation theory, the soot model aspect of the numerical model, and similar previous numerical studies. Chapter 4 entails the description, simulation procedure, and numerical results for both of the proposed models for a variety of coflow laminar diffusion flames. Chapter 5 concludes this body of work and finishes with recommendations for future work. 


\section{Chapter 2 - Numerical Model}

\subsection{Burner Configuration}

The numerical model that is used to conduct this research is an in-house FORTRAN combustion computational fluid dynamic model capable of simulating a variety of coflow laminar diffusion flames. It was formalized and published by Eaves [23], wherein the official name was given to be CoFlame, and is the result of the joint efforts of several Canadian universities and the National Research Council. A coflow burner is a thin walled cylindrical fuel tube contained within a larger thin walled cylindrical oxidizer tube and can be seen in Figure 2. This setup allows for a diffusion flame to occur wherein the oxidizer and fuel react at the flame front when undergoing combustion. Some of the changes that can be made to the model include: the burner dimensions, the fuel and oxidizer velocities, and the type of fuel being used.
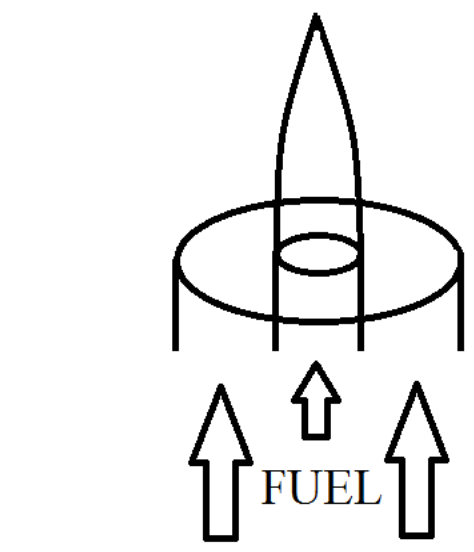

OXIDIZER

OXIDIZER

Figure 2: Coflow burner schematic.

Numerical simulations were conducted for both $C_{1}$ (methane) and $C_{2}$ (ethylene) fuels. The first simulated flame is from the works of Santoro et al. [15, 24], where experiments were conducted for ethylene/air coflow laminar diffusion flames with several different fuel and oxidizer velocities. Using laser extinction techniques, the soot volume fraction was measured at several different points along the wings and centerline of the ethylene/air flames. The burner 
that was used had an inner fuel tube diameter of $11.1 \mathrm{~mm}$ and had an outer oxidizer tube diameter of $101.6 \mathrm{~mm}$ with a wall thickness of $1 \mathrm{~mm}$ for both tubes. Of those flames seen in Table 1 , the one of particular interest was the second non-smoking flame (NSII) due to its prevalence in the literature and abundance of experimental data $[25,26,27,28]$. The NSII flame has a fuel velocity of $3.98 \mathrm{~cm} / \mathrm{s}$ and an oxidizer velocity of $8.9 \mathrm{~cm} / \mathrm{s}$. Additionally, the NSIl flame was selected to be the primary focus because of the fact that CoFlame was validated against the experimental data for the NSII flame [23].

Table 1: Santoro et al. [15, 24] Flow Conditions

\begin{tabular}{|c|c|c|c|c|c|}
\hline Experiment No. & Fuel & $\begin{array}{c}\text { Fuel Flow } \\
\text { Rate }\left(\mathrm{cm}^{3} / \mathrm{s}\right)\end{array}$ & $\begin{array}{c}\text { Velocity } \\
(\mathrm{cm} / \mathrm{s})\end{array}$ & $\begin{array}{c}\text { Air Flow } \\
\text { Rate }\left(\mathrm{cm}^{3} / \mathrm{s}\right)\end{array}$ & $\begin{array}{c}\text { Velocity } \\
(\mathrm{cm} / \mathrm{s})\end{array}$ \\
\hline $\begin{array}{c}1 \\
\text { (Non-Smoking I) }\end{array}$ & Ethylene & 2.30 & 2.38 & 713.3 & 8.90 \\
\hline $\begin{array}{c}2 \\
\text { (Non-Smoking II) }\end{array}$ & Ethylene & 3.85 & 3.98 & 713.3 & 8.90 \\
\hline $\begin{array}{c}3 \\
\text { (Incipient } \\
\text { Smoking) }\end{array}$ & Ethylene & 4.60 & 4.75 & 713.3 & 8.90 \\
\hline \begin{tabular}{c} 
(Smoking) \\
\hline
\end{tabular} & Ethylene & 4.90 & 5.05 & 1068.3 & 13.3 \\
\hline
\end{tabular}

Similarly, Lee et al. [29] conducted experiments for a methane/air coflow laminar diffusion flame using laser extinction techniques to measure soot volume fraction at several points along the wings and centerline. The burner that was used for the methane/air flame had the same dimensions as the burner that was used for the NSII flame. However, the inlet fuel velocity was $10.24 \mathrm{~cm} / \mathrm{s}$ whereas the inlet oxidizer velocity was $11.94 \mathrm{~cm} / \mathrm{s}$, which was different from the NSII flame. This methane/air flame was selected for the same reason as the NSII flame, which was because Eaves [23] validated CoFlame against this experimental data. 


\subsection{Governing Equations}

CoFlame is a fully coupled Navier-Stokes solver that solves elliptical conservation equations for mass, momentum, and energy. Furthermore, the conservation of species mass fraction is computed for the chemical species present in the gas phase as prescribed by the chemical kinetic mechanism. The chemical kinetic mechanism is a network of chemical reactions, which for this model occur in the gas phase. The governing equations are presented using cylindrical coordinates because of the axisymmetric nature a coflow laminar diffusion flame. These equations are presented in the CoFlame transcript [23].

Conservation of mass:

$$
\frac{1}{r} \frac{\partial}{\partial r}(r \rho v)+\frac{\partial}{\partial z}(\rho u)=0
$$

Where $r$ is the radial position, $\rho$ is the mixture density, $v$ is the radial velocity, $z$ is the axial position, and $u$ is the axial velocity.

\section{Conservation of axial momentum:}

$$
\begin{aligned}
\rho v \frac{\partial u}{\partial r}+\rho u \frac{\partial u}{\partial z} & \\
& =-\frac{\partial p}{\partial z}+\frac{1}{r} \frac{\partial}{\partial r}\left(r \mu \frac{\partial u}{\partial r}\right)+2 \frac{\partial}{\partial z}\left(\mu \frac{\partial u}{\partial z}\right)-\frac{2}{3} \frac{\partial}{\partial z}\left[\frac{\mu}{r} \frac{\partial}{\partial r}(r v)\right] \\
& -\frac{2}{3} \frac{\partial}{\partial z}\left(\mu \frac{\partial u}{\partial z}\right)+\frac{1}{r} \frac{\partial}{\partial r}\left(r \mu \frac{\partial v}{\partial z}\right)+\rho g_{z}
\end{aligned}
$$

Where $p$ is the pressure, $\mu$ is the dynamic viscosity, and $g_{z}$ is the acceleration due to gravity. 
Conservation of radial momentum:

$$
\begin{aligned}
\rho v \frac{\partial v}{\partial r}+\rho u \frac{\partial v}{\partial z} & \\
& =-\frac{\partial p}{\partial z}+\frac{2}{r} \frac{\partial}{\partial r}\left(r \mu \frac{\partial v}{\partial r}\right)+\frac{\partial}{\partial z}\left(\mu \frac{\partial v}{\partial z}\right)-\frac{2}{3} \frac{1}{r} \frac{\partial}{\partial z}\left[\mu \frac{\partial}{\partial r}(r v)\right] \\
& -\frac{2}{3} \frac{1}{r} \frac{\partial}{\partial r}\left(r \mu \frac{\partial u}{\partial z}\right)+\frac{\partial}{\partial z}\left(\mu \frac{\partial u}{\partial r}\right)-\frac{2 \mu v}{r^{2}}+\frac{2}{3} \frac{\mu}{r^{2}} \frac{\partial}{\partial r}(r v)+\frac{2}{3} \frac{\partial u}{\partial z}
\end{aligned}
$$

Conservation of energy:

$$
\begin{aligned}
c_{p}\left(\rho v \frac{\partial T}{\partial r}+\rho u\right. & \left.\frac{\partial T}{\partial z}\right) \\
& =\frac{1}{r} \frac{\partial}{\partial r}\left(r \lambda \frac{\partial T}{\partial r}\right)+\frac{\partial}{\partial z}\left(\lambda \frac{\partial T}{\partial z}\right)-\sum_{k=1}^{K K} h_{k} W_{k} \dot{\omega}_{k} \\
& -\sum_{k=1}^{K K}\left[\rho c_{p, k} Y_{k}\left(V_{k, r} \frac{\partial T}{\partial r}+V_{k, z} \frac{\partial T}{\partial z}\right)\right]-\rho c_{p, s} Y_{s}\left(V_{s, r} \frac{\partial T}{\partial r}+V_{s, z} \frac{\partial T}{\partial z}\right) \\
& -h_{s} W_{s} \dot{\omega}_{s}+Q_{r}
\end{aligned}
$$

Where $c_{p}$ is the mixture specific heat capacity at constant pressure, $T$ is the temperature, $\lambda$ is the mixture thermal conductivity, $h_{k}$ is the specific enthalpy of the $k^{\text {th }}$ species, $W_{k}$ is the molecular weight of the $k^{\text {th }}$ species, $\omega_{k}$ is the production rate of the $k^{\text {th }}$ species, $c_{p, k}$ is the specific heat capacity of the $k^{\text {th }}$ species, $Y_{k}$ is the mass fraction of the $k^{\text {th }}$ species, $c_{p, s}$ is the specific heat capacity of soot, $Y_{s}$ is the mass fraction of soot, $V_{s, r}$ is the soot radial diffusion velocity, $V_{s, z}$ is the soot axial diffusion velocity, $h_{s}$ is the specific enthalpy of soot, $W_{s}$ is the molecular weight of soot, and $Q_{r}$ is the radiative heat transfer by soot, $\mathrm{H}_{2} \mathrm{O}, \mathrm{CO}_{2}$, and $\mathrm{CO}$. 
Conservation of species mass fractions:

$$
\begin{gathered}
\rho v \frac{\partial Y_{k}}{\partial r}+\rho u \frac{\partial Y_{k}}{\partial z}=-\frac{1}{r} \frac{\partial}{\partial r}\left(r \rho Y_{k} V_{k, r}\right)-\frac{\partial}{\partial z}\left(\rho Y_{k} V_{k, z}\right)+W_{k} \dot{\omega}_{k} \\
(k=1,2, \ldots, K K)
\end{gathered}
$$

Where the range of values for $k$ is 1 to $K K$, the number of species included in the numerical model, which in the present work is 94 .

Furthermore, two transport equations are included for soot aggregate number density and soot primary particle number density. These equations are presented in the CoFlame transcript [23].

Conservation of soot aggregate number density

$$
\begin{aligned}
\rho v \frac{\partial N_{i}^{a}}{\partial r}+\rho u & \frac{\partial N_{i}^{a}}{\partial z} \\
& =\frac{1}{r} \frac{\partial}{r}\left(r \rho D_{i}^{a} \frac{\partial N_{i}^{a}}{\partial r}\right)+\frac{\partial}{\partial z}\left(\rho D_{i}^{a} \frac{\partial N_{i}^{a}}{\partial z}\right)-\frac{1}{r} \frac{\partial}{\partial r}\left(r \rho N_{i}^{a} V_{T_{s, r}}\right) \\
& -\frac{\partial}{\partial z}\left(\rho N_{i}^{a} V_{T_{s, z}}\right) \\
& +\rho\left(\left.\frac{\partial N_{i}^{a}}{\partial t}\right|_{n u}+\left.\frac{\partial N_{i}^{a}}{\partial t}\right|_{c o}+\left.\frac{\partial N_{i}^{a}}{\partial t}\right|_{s g}+\left.\frac{\partial N_{i}^{a}}{\partial t}\right|_{o x}+\left.\frac{\partial N_{i}^{a}}{\partial t}\right|_{f r}\right) \\
\quad(i=1,2, \ldots, 35) &
\end{aligned}
$$

Where $N_{i}^{a}$ is the aggerate number density, $D_{i}^{a}$ is the particle diffusion term, $V_{T s, r}$ is the radial thermophoretic velocity, $V_{T s, z}$ is the axial thermophoretic velocity, $n u$ is nucleation, co is PAH condensation, $s g$ is surface growth, ox is oxidation, and $f r$ is fragmentation. The range of values for $i$ is from 1 to 35, the number of bins for the soot sectional model. 
Conservation of soot primary particle number density

$$
\begin{aligned}
\rho v \frac{\partial N_{i}^{p}}{\partial r}+\rho u & \frac{\partial N_{i}^{p}}{\partial z} \\
& =\frac{1}{r} \frac{\partial}{\partial r}\left(r \rho D_{i}^{a} \frac{\partial N_{i}^{p}}{\partial r}\right)+\frac{\partial}{\partial z}\left(\rho D_{i}^{a} \frac{\partial N_{i}^{p}}{\partial z}\right)-\frac{1}{r} \frac{\partial}{\partial r}\left(r \rho N_{i}^{p} V_{T_{s, r}}\right) \\
& -\frac{\partial}{\partial z}\left(\rho N_{i}^{p} V_{T_{s, z}}\right) \\
& +\rho\left(\left.\frac{\partial N_{i}^{p}}{\partial t}\right|_{n u}+\left.\frac{\partial N_{i}^{p}}{\partial t}\right|_{c o}+\left.\frac{\partial N_{i}^{p}}{\partial t}\right|_{s g}+\left.\frac{\partial N_{i}^{p}}{\partial t}\right|_{o x}+\left.\frac{\partial N_{i}^{p}}{\partial t}\right|_{f r}\right) \\
& (i=1,2, \ldots, 35)
\end{aligned}
$$

Where $N_{i}^{p}$ is the soot primary particle number density.

Thermophoresis is the creation of a concentration gradient due to a thermal gradient being present. The hotter gas molecules will have higher velocities when colliding with colder gas molecules thus resulting in a net force towards the colder gas area. To account for the affects of thermophoresis, thermophoretic velocities need to be calculated which is given by [23]:

$$
V_{T_{s, x_{i}}}=-0.55 \frac{\mu}{\rho T} \frac{\partial T}{\partial x_{i}}\left(x_{i}=r, z\right)
$$

Several rates within the transport equations are needed in order to be able to solve the system of equations. These required rates will be presented in the following chapter that discusses the soot model. The variables of interest here are the rates for nucleation, condensation, surface growth, oxidation, and fragmentation.

\subsection{Radiation Model}

The radiation model in CoFlame uses the discrete ordinates method coupled with the statistical narrow-band correlated-k-based model to calculate radiative heat transfer [30]. The 
radiative transfer equation is integrated along a prescribed number of discrete ordinates to obtain a numerical solution. The equation is presented in cylindrical coordinates and is given by [23]:

$$
-\kappa I_{m, l}+\kappa I_{b}=\frac{\mu_{m, l}}{r} \frac{\partial r I_{m, l}}{\partial r}+\xi_{m, l} \frac{\partial I_{m, l}}{\partial z}-\frac{1}{r} \frac{\alpha_{m, l+\frac{1}{2}} I_{m, l+\frac{1}{2}}-\alpha_{m, l-\frac{1}{2}} I_{m, l-\frac{1}{2}}}{\omega_{m, l}}
$$

Where $m$ is the polar angle index from 1 to $\mathrm{M}$ (based on the number of ordinates selected), $l$ is the azimuthal angle index from 1 to $L(m)$ (based on the number of ordinates selected), $\mu$ and $\xi$ are directional cosines, $K$ is the spectral absorption coefficient, $I$ is the spectral intensity, $I_{B}$ is the spectral black-body intensity, $\alpha$ is a geometric constant [31], $\omega$ is a weighting function associated with the direction defined by $(m, l)$. The set of discrete ordinates is defined using $T_{3}$ quadrature for an axisymmetric cylinder [32], which simply defines a set of points for the integration. The intensities are calculated at the defined points and are related back to the cell center where the heat flux is computed.

\subsection{Boundary Conditions}

The boundary conditions are prescribed in [33] and a schematic can be seen in Figure 3.

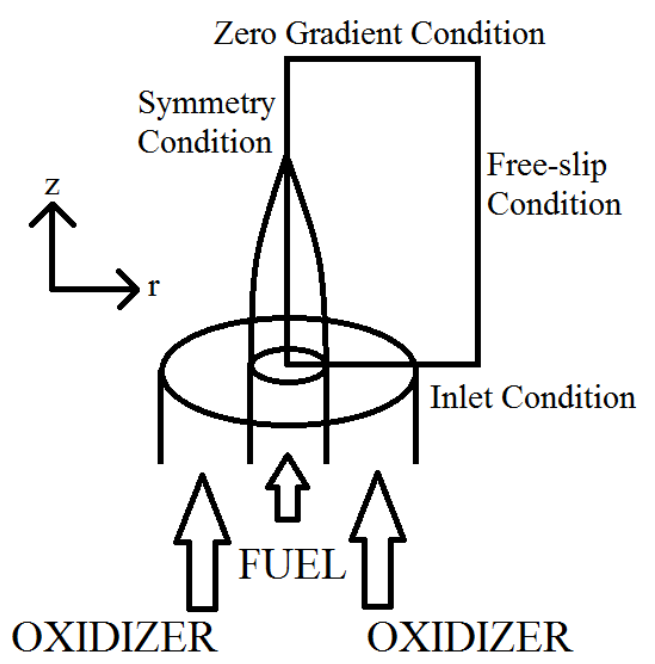

Figure 3: Coflow burner schematic with boundary conditions present. 
Inlet

Experiments used constant mass flow rates for both the fuel and air. Inlet velocities are assumed to have a flat profile and are calculated through the mass flow rates, ideal gas law, and burner dimensions.

$$
\begin{gathered}
u=u_{\text {fuel }}, Y_{\text {fuel }}=1 r \leq 5.55 \mathrm{~mm} \\
u=0, Y_{k}=05.55 \leq r \leq 6.55 \mathrm{~mm} \\
u=u_{\text {air }}, Y_{O_{2}}=0.231, Y_{N_{2}}=0.769 r \geq 6.55 \mathrm{~mm} \\
v=0, N_{i}^{a}=0, N_{i}^{p}=0, T=300 \mathrm{~K}
\end{gathered}
$$

Axis of symmetry

The following zero-gradient conditions are applied to the axis of symmetry.

$$
\frac{\partial u}{\partial r}=0, v=0, \frac{\partial T}{\partial r}=0, \frac{\partial Y_{k}}{\partial r}=0, \frac{\partial N_{i}^{p}}{\partial r}=0, \frac{\partial N_{i}^{a}}{\partial r}=0
$$

\section{Outer radial boundary}

The following zero-gradient conditions are applied as well as the species mass fractions of ambient air are imposed.

$$
u=0, v=0, \frac{\partial T}{\partial r}=0, Y_{O_{2}}=0.231, Y_{N_{2}}=0.769, \frac{\partial N_{i}^{p}}{\partial r}=0, \frac{\partial N_{i}^{a}}{\partial r}=0
$$

\section{Outlet}

The following zero-gradient conditions are imposed. 


$$
\dot{m}_{\text {outflow }}=\dot{m}_{\text {inlet }}, \frac{\partial v}{\partial z}=0, \frac{\partial T}{\partial z}=0, \frac{\partial Y_{k}}{\partial z}=0, \frac{\partial N_{i}^{p}}{\partial z}=0, \frac{\partial N_{i}^{a}}{\partial z}=0
$$

\subsection{Numerical Method}

The computational domain that was used to conduct this research was $30.226 \mathrm{~cm}$ in the axial direction and $4.206 \mathrm{~cm}$ in the radial direction. The domain was decomposed into 400 control volumes axially and 150 control volumes radially. A non-uniform mesh was employed to reduce computational time as the largest variable gradients occur in the flame region where the majority of chemical reactions occur. The finest control volumes in the mesh were $0.027 \mathrm{~cm}$ in the axial direction and $0.0125 \mathrm{~cm}$ in the radial direction. For the axial direction, the stretch rate factor was set to 1.05 and started after an axial position of $8.8 \mathrm{~cm}$. The stretch rate factor multiples the previous axial control volume spacing by 1.05 to obtain the spacing for the subsequent control volume. For the radial direction, the stretch rate factor was set to 1.038 after the $90^{\text {th }}$ control volume. Due to computational intensity, a mesh sensitivity study was not carried out in a classical sense where the computational domain is held constant while the spacing of the control volumes is reduced until a grid independent solution is obtained. However, as this flame is wellcharacterized and studied by many researchers guidelines have been established by the scientific community, who contribute to the the International Sooting Flame Workshop [34]. For example, it is suggested that the computational domain for the NSII flame be $12 \mathrm{~cm}$ axially, $4.75 \mathrm{~cm}$ radially, and contain at least 15000 control volumes. In the present work, care was taken to exceed each of these parameters.

The solution procedure is presented in the CoFlame transcript [23]. To handle pressure and velocity, a staggered mesh as seen in Figure 4, with a semi-implicit discretization scheme is employed. The axial velocity control volumes are offset vertically while the radial velocity control 
volumes are offset horizontally from the pressure control volumes. Using the pressure control volume layout, conservation equations are solved in the same manner for species, energy, and soot volume fraction. Power law schemes are used to discretize convective terms while diffusive terms are discretized with a second-order difference scheme [35]. Using the chemical kinetic mechanism, chemical reaction rates and thermal properties of gaseous species are obtained [36, 37]. Using the transport properties library, several transport properties are calculated such as mixture average viscosities, conductivities, and diffusion coefficients.

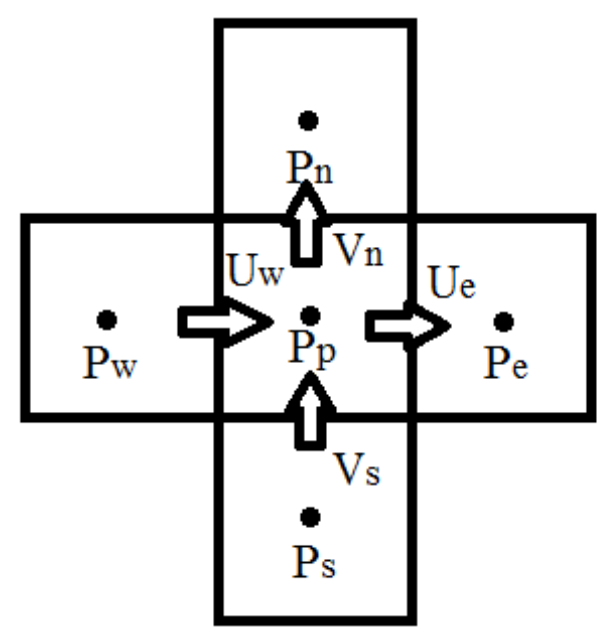

Figure 4: Staggered mesh where the velocity components are stored at the cell faces of the pressure control volumes.

From an arbitrary start point, pseudo transient continuation is employed for convergence [38]. Initially, the momentum and pressure correction equations are solved globally. A linear system coupling each of the species conservation equations is solved at each control volume to solve for the gaseous species. This is done to deal with the stiffness of the system and speedup convergence by using the tri-diagonal matrix algorithm. The sectional transport equations are solved in the same fashion after species has been solved. Lastly, the energy equation is solved globally, and the temperature field is updated. A convergence check is carried out wherein if the 
change in independent variables between timesteps is one thousandth of it previous value [33], the solution is deemed to have converged.

Due to the computational intensity, parallelization techniques have been employed to accelerate solution generation. Strip-domain decomposition is used wherein the computational domain is decomposed such that the number of processors is equal to the number of control volumes in the axial direction, which represents one subdomain. Thus, the number of subdomains that need to be solved in order to generate a solution for the entire domain is equal to the number of control volumes in the radial direction. The computations were conducted on the Niagara cluster of the SciNet HPC Consortium located at the University of Toronto. As previously mentioned, the computational domain used for this body of research was $400 \times 150$ control volumes. Thus, when submitting jobs to the queue, 400 processors were requested. 


\section{Chapter 3 - Soot Formation Theory}

\subsection{Gas Phase Chemistry}

Soot formation begins with the incomplete combustion of hydrocarbon fuel, where molecules in the gas phase react at high temperatures. These molecules participate in various reactions that involve species such as $\mathrm{C}_{2} \mathrm{H}_{2}, \mathrm{C}_{4} \mathrm{H}_{\mathrm{i}}, \mathrm{C}_{5} \mathrm{H}_{5}$, and $\mathrm{CH}_{3}[39,40,41,42]$ to form PAHs that will potentially become soot. Thus, one of the most important elements in any combustion numerical model is the chemical kinetic mechanism, as it prescribes those chemical reactions that are considered to be occurring in a simulation. In current literature, several mechanisms are commonly used with each having their own benefits and drawbacks. For example, having fewer species and reactions leads to faster compute times at the cost of accuracy in determining certain

characteristics. A previous study compared three kinetic mechanisms by Appel et al. [13], Marinov et al. [14], and Slavinskaya and Frank [43] based on varying PAH growth mechanisms. As a result of that body of work [11] and other studies [10, 12], the present numerical model uses the Slavinskaya and Frank [43] mechanism with some modifications described in the cited works. The aforementioned studies will be discussed in greater detail later in this chapter.

The chemical kinetic mechanism originally developed by Slavinskaya and Frank [43] was designed to predict PAH formation and their growth up to five aromatic rings. Furthermore, this kinetic mechanism consists of 93 species and 729 reactions and was intended for simulating methane and ethane laminar premixed flames. In order to reduce global reactions, some of the multistep sequences in aromatic growth were lumped together. The kinetic model consists of three sub mechanisms: methane oxidation, ethylene oxidation, and PAH formation up to 5-ring molecules. Based off both experimental and theoretical work, five PAH sub mechanisms were 
generated. Those sub mechanisms were: HACA mechanism with different small radicals applied for aromatic molecular activation, hydrogen atom migration, possible free radical addition schemes, cyclopentadienyl moiety in aromatic ring formation, and reactions between aromatic radicals and molecules. The results of 12 different groups studying laminar premixed flames at different $\mathrm{C} / \mathrm{O}$ ratios, pressures and under shock tube conditions were used to compare numerical results to, using the new kinetic mechanism. The mechanism had shown reasonably good agreement with flame speeds, concentration profiles of small molecules and radicals, medium size and high molecular mass rings and soot volume fractions [43]. The first major finding was that the main reaction routes could be broken down into either low temperature $(<1550 \mathrm{~K})$ steps or high temperature ( $>1550 \mathrm{~K})$ steps [43]. The high temperature steps were determined to be the main contributors to PAH formation [43]. Secondly, it was determined that large PAH molecules have three main and equally important reactions: HACA mechanism, reactions between aromatic molecules/radicals and small molecules, and reactions between aromatic molecules and aromatic radicals [43].

\subsection{Soot Inception}

Soot inception is defined as the point in which a soot particle is generated. This process accounts for the beginning of the transition of carbon matter from the gas phase to the solid phase. The mechanisms that are responsible for soot inception are not fully understood in the literature because of the complexity, resulting in a variety of hypothesises. One of the proposed pathways is through the generation of a structure similar to a fullerene ball [44]. However, the issue with this mechanism is that it relies on surface growth, which is known to not be able to account for all soot inception on its own [16]. The second mechanism is through chemical 
reactions that result in cross linked structures and the last is through the dimerization of large PAHs [45].

The contribution of each of these mechanisms is unknown and due to this uncertainty, assumptions must be made in any numerical model. The numerical model prescribes the rate of soot inception via only PAH dimerization in the free molecular collision regime with quantitative success [46]. Dimerization is the collision of two identical molecules that under the right conditions will result in an effective sticking together. To elaborate further, the sticking of these molecules could potentially be prescribed through either physical or chemical bonding. The community has yet to reach a uniform understanding of the type of bonding that would occur during the physical dimerization of these PAHs. However, assumptions can be made to act as preliminary steps towards the generation of a fundamental model, such as the manner in which these PAHs bond together after colliding. The governing equation for dimerization in the present model is given by [46]:

$$
\begin{gathered}
\left.\frac{\partial N_{1}^{a}}{\partial t}\right|_{n u}=\left.\frac{\partial N_{1}^{p}}{\partial t}\right|_{n u}=\beta \sqrt{\frac{8 \pi k_{B} T}{\mu_{A B}}}\left(r_{A}+r_{B}\right)^{2} A_{V}^{2}[A][B] \\
\left.\frac{\partial N_{1}^{a}}{\partial t}\right|_{n u}=\left.\frac{\partial N_{1}^{p}}{\partial t}\right|_{n u}=0, i=2,3, \ldots, 35
\end{gathered}
$$

Where $B$ is the collision efficiency, $k_{B}$ is the Boltzmann constant, $A_{V}$ is Avogadro's number, $\mu_{A B}$ is the collisional reduced mass for the two-colliding species, $r_{A}$ and $r_{B}$ are the radii of the twocolliding species, and $[A]$ and $[B]$ are the concentrations of the two-colliding species [46]. The collisional reduced mass converts the two particles into a single equivalent mass. This new mass 
passes through the center of the rotational axis and a distance from that axis is calculated to keep the moment of inertia constant.

A sectional soot model divides soot mass in different sections logarithmically based on mass. Inception is the first step in the lifetime of a soot particle and thus is calculated only for the

first soot section. Furthermore, the species that participate in soot inception via dimerization in the present model are benzo(a)pyrene (BAPYR), secondary benzo(a)pyrenyl (BAPYRS), and benzo(ghi)fluoranthene (BGHIF) [46]. Of all the terms present in the above equation, the collision efficiency is the only term that does not have a functional form or a fundamentally derived constant. It is a unitless term that represents the portion of collisions that will result in effective sticking together. A constant value of $1 \mathrm{e}-4$ is used as prescribed in the literature [46].

\subsection{Coagulation}

Aerosol particles undergo many collisions due to their random motion and eventually, under the right conditions, these particles with aggregate together to form larger clusters. In terms of soot modelling, the same holds true for soot primary particles and soot aggregates. The present model uses the entire Knudsen number regime to calculate coagulation terms based on the collision kernel of soot aggregates. A coagulation efficiency is set to 0.2 as according to the literature [38]. The equations that prescribe the coagulation terms for primary particles and aggregates in the present model are given by $[47,48]$ :

$$
\left.\frac{\partial N_{i}^{a}}{\partial t}\right|_{c o}=\sum_{m_{i-1} \leq m_{j}+m_{-} \leq \leq m_{i+1}}^{k \leq j \leq i}\left(1-\frac{\delta_{j, k}}{2}\right) \eta \beta_{j, k} \xi_{j, k} N_{j}^{a} N_{k}^{a}-N_{i}^{a} \sum_{k=1}^{35} \beta_{i, k} \xi_{i, k} N_{k}^{a}
$$




$$
\left.\frac{\partial N_{i}^{p}}{\partial t}\right|_{c o}=\sum_{m_{i-1} \leq m_{j}+m_{-} \leq \leq m_{i+1}}^{k \leq j \leq i}\left(1-\frac{\delta_{j, k}}{2}\right) \eta \eta_{p, i} \beta_{j, k} \xi_{j, k} N_{j}^{a} N_{k}^{a}-\eta_{p, i} N_{i}^{a} \sum_{k=1}^{35} \beta_{i, k} \xi_{i, k} N_{k}^{a}
$$

Where $m_{\mathrm{i}}$ is the representative mass of the $i^{\text {th }}$ section aggregate, $\delta$ is the Kronecker delta function, $B_{j, k}$ is the collision kernel of two aggregates in the $j^{\text {th }}$ and $k^{\text {th }}$ sections, $\eta_{p, i}$ is the number of primary particles per aggregate in the $i^{\text {th }}$ section, and $\xi_{j, k}$ is the coagulation efficiency of two aggregates in the $j^{\text {th }}$ and $k^{\text {th }}$ sections.

$$
\eta=\left\{\begin{array}{l}
\frac{m_{i+1}-\left(m_{j}+m_{k}\right)}{m_{i+1}-m_{i}} \text { if } m_{i} \leq m_{j}+m_{k} \leq m_{i+1} \\
\frac{m_{i-1}-\left(m_{j}+m_{k}\right)}{m_{i-1}-m_{i}} \text { if } m_{i-1} \leq m_{j}+m_{k} \leq m_{i}
\end{array}\right.
$$

Where $\eta$ ensures the number and mass of aggregates are conserved for the newly formed mass by weighing it into two adjacent sections.

$$
\eta_{p}=\frac{m_{i}}{m_{j}+m_{k}}\left(\eta_{p, j}+\eta_{p, i}\right)
$$

Where $\eta_{p}$ ensures primary particle size and number are conserved by assigning primary particles into two adjacent sections.

The collision kernel in the present model is given by [49]:

$$
\beta_{j, k}=4 \pi R_{a b s}\left(D_{j}+D_{k}\right) f_{D}
$$

Where $R_{a b s}$ is the absorbing sphere cluster radius, $D_{j}$ and $D_{k}$ are the diffusion coefficients for soot particles in the $j^{\text {th }}$ and $k^{\text {th }}$ sections, and $f_{D}$ is the transition regime correction factor.

The diffusion coefficients for both free molecular and continuum regimes in the present model are given by: 


$$
D=\frac{k_{B} T C_{c}(K n)}{3 \pi \mu d_{m}}
$$

Where $D$ is the diffusion coefficient, $d_{m}$ is the mobility diameter, and $C_{c}(K n)$ is the Cunningham slip correction factor.

The Cunningham slip correction factor in the present model is given by [50]:

$$
C_{c}(K n)=1+1.612 K n
$$

The Knudsen number in the present model is given by:

$$
K n=\frac{2 \lambda_{m f p}}{d_{m}}
$$

Where $\lambda_{m f p}$ is the mean free path of the gas and is taken to be as air.

The transition regime between free molecular and continuum regimes requires a correction factor that in the present model is given by:

$$
f_{D}=\frac{1+K n_{D}}{1+2 K n_{D}\left(1+K n_{D}\right)}
$$

The diffusion Knudsen number that describes the transition between continuum and free molecular diffusion in the present model is given by:

$$
\begin{gathered}
K n_{D}=\frac{\lambda_{m f p, 12}}{R_{a b s}} \\
\lambda_{m f p, 12}=\frac{D_{j}+D_{k}}{\sqrt{\frac{2 k_{B} T}{\pi}\left(\frac{1}{m_{j}}+\frac{1}{m_{k}}\right)}}
\end{gathered}
$$

Where $m_{j}$ and $m_{k}$ is the mass of soot aggregate in the $j^{\text {th }}$ and $k^{\text {th }}$ sections.

The mobility diameter in the present model is given by: 


$$
d_{m}=\left\{\begin{array}{c}
2 r_{p} n_{p}^{0.43} \text { free molecular regime } \\
2 R_{f}\left(\frac{D_{f}-1}{2}\right)^{0.7} \text { continuum regime }
\end{array}\right.
$$

Where $r_{p}$ is the primary particle radius, $n_{p}$ is the number of primary particles in the aggregate, $D_{f}$ is the fractal dimension, and $R_{f}$ is the outer radius of an aggregate.

The outer radius of an aggregate requires a volume filling factor, which set to 1.43 [51] as according to the literature and in the present model is given by:

$$
R_{f}=r_{p}\left(f n_{p}\right)^{\frac{1}{D_{f}}}
$$

Where $f$ is the volume filling factor.

\subsection{Soot Surface Reactions}

\subsubsection{Hydrogen-Abstraction-Carbon Addition and Oxidation}

Soot surface reactivity was introduced by Frenklach and Wang [9] and is defined as the portion of active sites on the surface of a soot particle available for reaction. As per its definition, its value must be in the range of 0 to 1 as it is a fraction of the total number of sites. Moreover, it is either kept as a constant value $[10,11,12]$ or more recently has been given a functional form $[52,53,54]$ in many numerical models. The framework for the HACA mechanism and $\mathrm{O}_{2}$ driven oxidation rely on the theory of soot surface sites to function.

The HACA mechanism operates through the removal of a hydrogen atom from the surface of a soot particle followed by the addition of an acetylene molecule. Through this process, carbon mass is added to the soot particle and it becomes larger. Surface reactivity comes into play here as not all sites may be active for the removal of hydrogen and subsequent addition of acetylene. The mechanism was implemented into soot modelling based off the assumption that all forms of 
carbonaceous materials experience growth through the HACA reaction sequence at high temperatures [9]. The governing equation for HACA surface growth is given by [9]:

$$
\text { HACA Surface Growth }=k_{g, s} C_{g} \propto \chi_{s} S_{i} N_{i}
$$

Where $k_{g, s}$ is the per-site rate coefficient, $\mathrm{C}_{g}$ is the concentration of colliding gaseous species $\mathrm{C}_{2} \mathrm{H}_{2}$, $\alpha$ is the fraction of surface sites available for reaction for a given reaction, $\chi_{s}$ is the number density of surface sites, $S_{i}$ is the surface of the $i^{\text {th }}$ particle, and $N_{i}$ is the number density of the $i^{\text {th }}$ particle.

There are 2 species that are considered to participate in the oxidation of soot and those are $\mathrm{O}_{2}$ and $\mathrm{OH}$, which form the same product through different means. Using the same theory of soot surface sites, $\mathrm{O}_{2}$ strips carbon mass from the surface of a soot particle to form $\mathrm{CO}$. In contrast, $\mathrm{OH}$ driven oxidation functions in the free molecular collision regime to form carbon monoxide through effective collisions between $\mathrm{OH}$ molecules and soot particles. The same framework is used here to handle collisions in the free molecular collision regime as is done for soot inception. Thus, the present model prescribes an $\mathrm{OH}$ collision efficiency of 0.13 according to the literature [55]. Table 2 summarizes the 6 reactions of the HACA and oxidation model developed by Frenklach and coworkers $[9,13]$ that is used in the present numeric model.

Table 2: HACA and Oxidation Reaction Sequence

\begin{tabular}{|c|c|c|c|c|}
\hline No. & Reaction & $\mathbf{A}\left(\mathbf{c m}^{\mathbf{3}} \mathbf{m o l}^{-1} \mathbf{s}^{-1}\right)$ & $\mathbf{b}$ & $\mathbf{E}_{\mathbf{a}}$ (kcal/mol) \\
\hline 1 & $\mathrm{C}_{\text {soot }}-\mathrm{H}+\mathrm{H} \leftrightarrow \mathrm{C}_{\text {soot }}+\mathrm{H}_{2}$ & $4.2 \times 10^{13}$ & 0 & 13 \\
\hline 2 & $\mathrm{C}_{\text {soot }}-\mathrm{H}+\mathrm{OH} \leftrightarrow \mathrm{C}_{\text {soot }}+\mathrm{H}_{2} \mathrm{O}$ & $1.0 \times 10^{10}$ & 0.73 & 1.43 \\
\hline 3 & $\mathrm{C}_{\text {soot }}+\mathrm{H} \leftrightarrow \mathrm{C}_{\text {soot }}-\mathrm{H}$ & $2.0 \times 10^{13}$ & 0 & 0 \\
\hline 4 & $\mathrm{C}_{\text {soot }}+\mathrm{C}_{2} \mathrm{H}_{2} \leftrightarrow \mathrm{C}_{\text {soot }}-\mathrm{H}+\mathrm{H}$ & $8.0 \times 10^{7}$ & 1.56 & 3.8 \\
\hline 5 & $\mathrm{C}_{\text {soot }}+\mathrm{O}_{2} \leftrightarrow 2 \mathrm{CO}+$ product & $2.2 \times 10^{12}$ & 0 & 7.5 \\
\hline 6 & $\mathrm{C}_{\text {soot }}+\mathrm{OH} \leftrightarrow \mathrm{CO}+$ product & \multicolumn{3}{|c|}{$\mathrm{YoH}_{\mathrm{OH}}=0.13$} \\
\hline
\end{tabular}




\subsubsection{Polycyclic Aromatic Hydrocarbon Condensation}

$\mathrm{PAH}$ condensation accounts for the addition of carbon mass through the effective collisions of PAHs and soot particles. In contrast to the free molecular collision regime that soot inception and $\mathrm{OH}$ driven oxidation operates in, $\mathrm{PAH}$ condensation operates in the transition and continuum collision regime. A collision efficiency of 1.0 is prescribed as according to the literature [38]. The total surface growth and oxidation rates for both primary particles and aggregates in the present model are given by:

$$
\begin{aligned}
& \left.\frac{\partial N_{i}^{a}}{\partial t}\right|_{s g}=\left\{\begin{array}{c}
-\frac{I_{g, i}}{m_{i+1}-m_{i}} \text { if } i=1 \\
\frac{I_{g, i-1}}{m_{i}-m_{i-1}}-\frac{I_{g, i}}{m_{i+1}-m_{i}} \text { if } i=2, \ldots, S N-1 \\
\frac{I_{g, i-1}}{m_{i}-m_{i-1}} \text { if } i=S N
\end{array}\right. \\
& \left.\frac{\partial N_{i}^{p}}{\partial t}\right|_{s g}=\left\{\begin{array}{c}
-\frac{I_{g, i}}{m_{i+1}-m_{i}} \eta_{p, i} \text { if } i=1 \\
\frac{I_{g, i-1}}{m_{i}-m_{i-1}} \eta_{p, i-1}-\frac{I_{g, i}}{m_{i+1}-m_{i}} \eta_{p, i} \text { if } i=2, \ldots, S N-1 \\
\frac{I_{g, i-1}}{m_{i}-m_{i-1}} \eta_{p, i-1} \text { if } i=S N
\end{array}\right.
\end{aligned}
$$

Where $I_{g, i}$ is the total surface growth rate of PAH condensation and HACA for the $i^{\text {th }}$ section.

$$
\left.\frac{\partial N_{i}^{a}}{\partial t}\right|_{o x}=\left\{\begin{array}{c}
-\frac{I_{o x, i+1}}{m_{i+1}-m_{i}}+\frac{I_{o x, i}}{m_{i}} \text { if } i=1 \\
\frac{I_{o x, i}}{m_{i}-m_{i-1}}-\frac{I_{o x, i+1}}{m_{i+1}-m_{i}} \text { if } i=2, \ldots, S N-1 \\
\frac{I_{o x, i}}{m_{i}-m_{i-1}} \text { if } i=S N
\end{array}\right.
$$




$$
\left.\frac{\partial N_{i}^{a}}{\partial t}\right|_{o x}=\left\{\begin{array}{c}
-\frac{I_{o x, i+1}}{m_{i+1}-m_{i}} \eta_{p, i+1}+\frac{I_{o x, i}}{m_{i}} \eta_{p, i} \text { if } i=1 \\
\frac{I_{o x, i}}{m_{i}-m_{i-1}} \eta_{p, i}-\frac{I_{o x, i+1}}{m_{i+1}-m_{i}} \eta_{p, i+1} \text { if } i=2, \ldots, S N-1 \\
\frac{I_{o x, i}}{m_{i}-m_{i-1}} \eta_{p, i} \text { if } i=S N
\end{array}\right.
$$

Where $I_{o x, i}$ is the oxidation rate for the $i^{\text {th }}$ section.

\subsection{Fragmentation}

The present model considers only oxidation driven fragmentation wherein soot aggregates are broken down into smaller aggregates. This occurs only after a collision happens with enough impact energy to break apart the bonds that are holding the aggregate together. Fragmentation is a complex phenomenon and much of it is still unknown [56] such as the pattern in which in the fragmentation occurs. A fragmentation pattern of 1:1 is used only on aggregates and not primary particles. The governing equation in the present model is given by $[38,56]$ :

$$
\begin{gathered}
\left.\frac{\partial N_{i}^{a}}{\partial t}\right|_{f r}=\left\{\begin{array}{c}
\Gamma_{i, i+1} S_{i+1} N_{i+1}^{a} \text { if } i=1 \\
\left(\Gamma_{i, i}-1\right) S_{i} N_{i}^{a}+\Gamma_{i, i+1} S_{i+1} N_{i+1}^{a} \text { if } i=2, \ldots, S N-1 \\
\left(\Gamma_{i, i}-1\right) S_{i} N_{i}^{a} \text { if } i=S N
\end{array}\right. \\
\left.\frac{\partial N_{i}^{p}}{\partial t}\right|_{f r}=\left\{\begin{array}{c}
\Gamma_{i, i+1} S_{i+1} N_{i+1}^{a} n_{P A H, i+1} \\
f_{S} \text { if } i=1 \\
\left(\Gamma_{i, i}-1\right) S_{i} N_{i}^{a} n_{P A H, i}+\frac{\Gamma_{i, i+1} S_{i+1} N_{i+1}^{a} n_{p, i+1}}{f_{S}} \text { if } i=2, \ldots, S N-1 \\
\left(\Gamma_{i, i}-1\right) S_{i} N_{i}^{a} n_{P A H, i}
\end{array}\right.
\end{gathered}
$$

Where $\Gamma_{i, i}$ and $\Gamma_{i, i+1}$ are the breakage distribution functions and $S_{i}$ is the fragmentation rate of the $i^{\text {th }}$ section.

$$
\Gamma(i, i)=\frac{f_{s}-2}{f_{s}-1} \Gamma_{i, i+1}=\frac{f_{s}}{f_{s}-1}
$$




$$
S_{i}=A\left(n_{p, 1}\right)^{\frac{1}{D_{f}}}
$$

Where $A$ is a coefficient that governs the overall fragmentation rate.

The overall fragmentation rate is taken to be a first order equation as an approximation. The constant is set to be $1 \times 10^{5}[38,56]$.

$$
A=C r_{o x, s}
$$

Where $r_{o x, s}$ is the rate of removal of soot mass per unit soot surface area and C is a constant.

\subsection{Previous Numerical Studies}

\subsubsection{Influence of the Chemical Kinetic Mechanism}

The kinetic mechanism developed by Slavinskaya and Frank [43] was implemented by Dworkin et al. [11] wherein the mechanism was applied to a parallelized coflow laminar diffusion combustion computational fluid dynamic model, which was a precursor to CoFlame. Validation for the model had been done for zero and one-dimensional premixed flame systems and had been updated for a coflow burner configuration for a sooting ethylene/air diffusion flame [24]. The objective of the work conducted by Dworkin et al. [11] was to compare the performance of the updated kinetic mechanism of Slavinskaya and Frank [43] to the Appel et al. [13] and Marinov et al. [14] kinetic mechanisms. The first key finding of the analysis was that fuel oxidation chemistry had been essentially the same for the three mechanisms; however, the ways through which PAH growth occurred was considerably different [11].

The results showed that the updated Slavinskaya and Frank [43] kinetic mechanism was capable of predicting a maximum soot volume fraction close to the experimental maximum of $9.5 \mathrm{ppm}$ when surface reactivity was set to 0.078. The Appel et al. [13] kinetic mechanism 
predicted a maximum soot volume fraction of $9 \mathrm{ppm}$ when surface reactivity was set to 1 . The Marinov et al. [14] kinetic mechanism performed the worst as no value for surface reactivity in the range of 0 to 1 was capable of predicting a maximum soot volume fraction close to the experimental value.

One of the most significant improvements that the updated Slavinskaya and Frank mechanism provided was in its prediction of the soot volume fraction along the centerline. Only the updated Slavinskaya and Frank mechanism was capable of predicting the correct order of magnitude of the soot volume fraction along the centerline as seen in Figure 5.

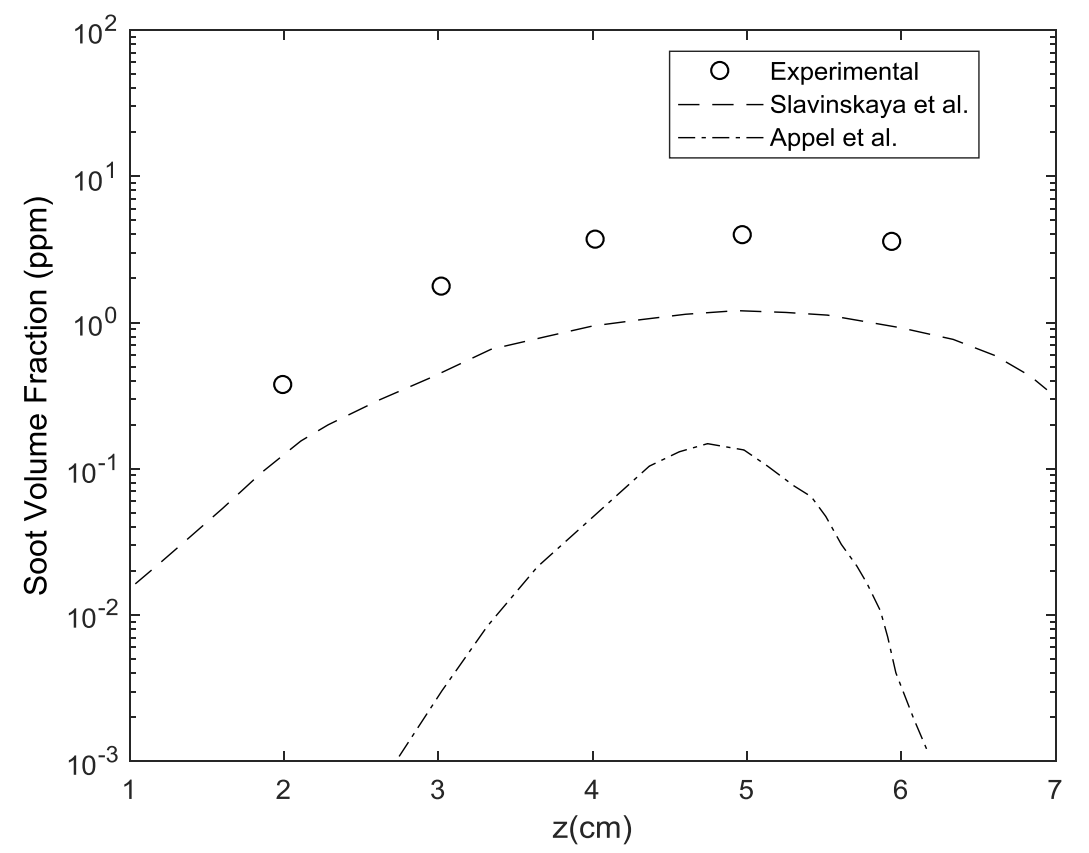

Figure 5: Predicted soot volume fraction as a function of height above the burner along the centerline for the Slavinskaya and Frank [43] mechanism, Appel et al. mechanism [13], and experimental data along the centerline of the NSII flame [24]. Reproduced from Dworkin et al. [11].

Current literature suggests that the soot volume fraction along the centerline of a flame is closely related to inception rates whereas the wings are dominated by surface growth through the HACA mechanism [10]. The improvement along the centerline was attributed to the increased prediction of benzene and pyrene concentrations, which are known to be soot 
precursor species. The enhanced PAH growth pathways allowed for this to occur. Simultaneously, the acetylene concentrations were not significantly different between the two mechanisms, which indicated that the HACA mechanism was not responsible for this soot mass growth.

The application of the Slavinskaya and Frank [43] mechanism was not only able to provide better predictions of soot precursor species and the soot volume fraction along the centerline but did so without having an unrealistic surface reactivity [11]. The Appel et al. [13] mechanism required a surface reactivity to be set to unity in order to predict a maximum soot volume fraction close to the experimental value, as the surface growth had to account for soot inception deficiencies [11].

The importance of these findings with regards to the present thesis is to understand how changes in the routes to the formation of PAHs influence the behaviour of the NSII flame and how those changes were tested. The present thesis aims to achieve a similar concept by changing the current routes to soot inception, which can yield the same effects as changing the routes to PAH formation. Moreover, Dworkin et al. [11] demonstrated how surface reactivity was iteratively computed in the numerical model in order to achieve experimental values. The importance of this iterative scheme is to understand how influential surface reactivity can be on the formation of soot and how it could be used to account for inception deficiencies within the numerical model. Lastly, understanding the centerline behaviour of the NSII flame with regards to which precursor species influence soot mass growth was important. The present thesis aims to improve centerline prediction, which is only achievable if the behaviour of the centerline can be understood. 


\subsubsection{Application to Partially Premixed Systems}

After applying the Slavinskaya and Frank [43] mechanism to a coflow laminar diffusion ethylene/air flame in the work of Dworkin et al. [11], the same solver was then used to test the prediction capabilities for a different flame system in [10]. Instead of a non-premixed flame, it

was two laminar partially premixed ethylene/air flames $[57,58]$. One of the key parameters to these simulations was the use of primary equivalence ratio $(\phi)$, which was defined as the ratio of the primary air flowrate that is required for complete combustion to the actual primary air flow rate [10]. The two experimental datasets used a range of different primary equivalence ratios, but both contained a case that represented a diffusion flame.

The three main processes that influence soot growth are inception, surface growth through the HACA mechanism, and PAH condensation [10]. These processes were separated into two categories. One category was PAH related processes, which included PAH condensation and inception. The other was acetylene related processes and contained only the HACA mechanism. The reason behind this treatment was to observe the effects that PAHs had on soot mass growth separately from the influence of acetylene in different regions of the flame. The numerical results of that analysis can be seen in Figure 6 for the centerline of the ethylene/air flame. The significance of this analysis, for the present thesis, is with regards to the results for the diffusion flame. It can be seen that for the centerline of the ethylene flame, both PAH and acetylene related processes are influential in formation of soot as neither dominate along the centerline. 


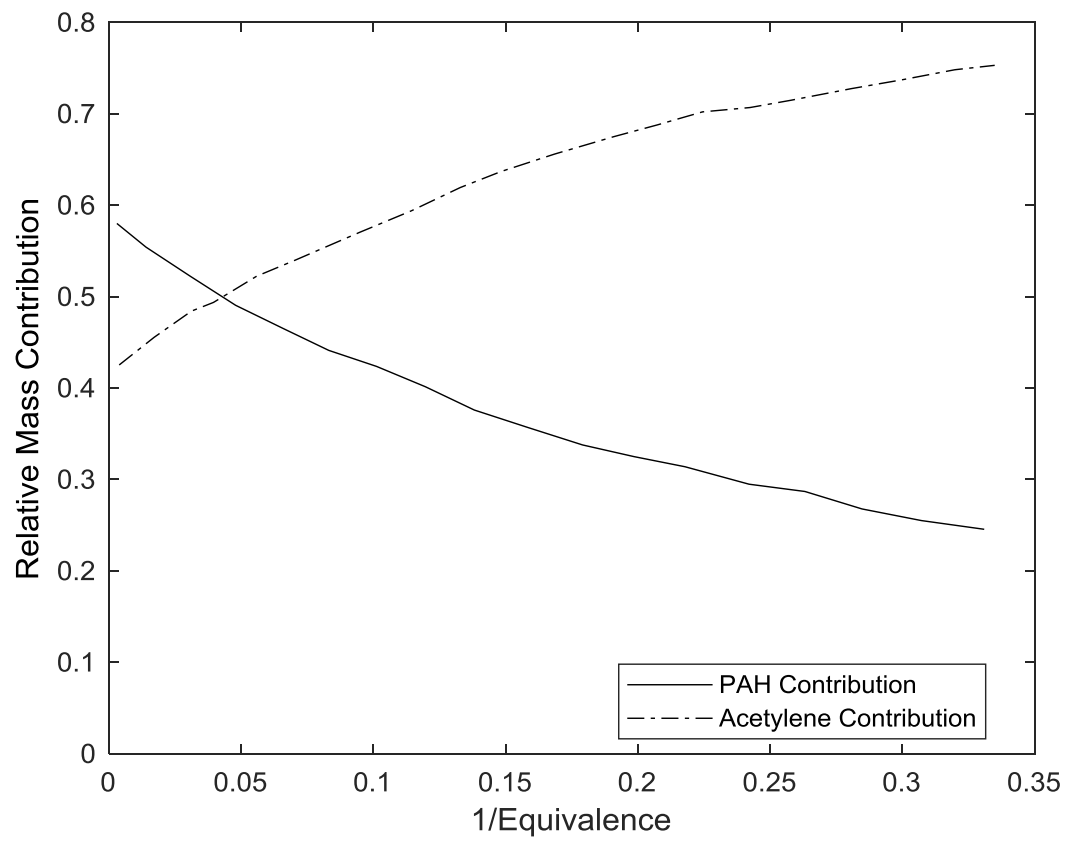

Figure 6: Relative mass contribution for the McEnally and Pfefferle [12] ethylene partially premixed flame along the centerline for different equivalence ratios. Reproduced from Chernov et al. [10].

Furthermore, although neither process was dominant in soot mass growth, it was known in the literature [10] that the soot yield was closely related to the inception rate. This correlation between the soot yield and the inception rate can be seen in Figure 7 for the ethylene/air flame. This indicates that inception is a critical component in the formation of soot along the centerline despite neither process being dominant. 


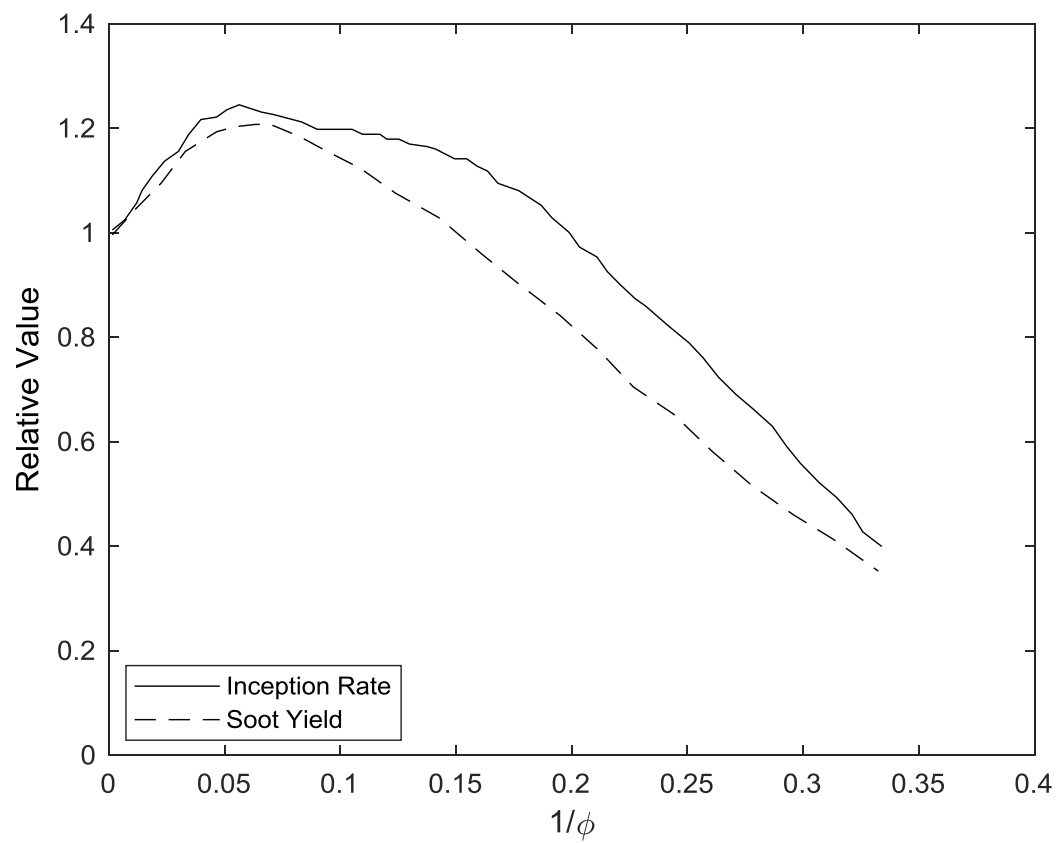

Figure 7: Relative value for McEnally and Pfefferle [58] ethylene partially premixed flame along the centerline for different equivalence ratios. Reproduced from Chernov et al. [10].

PAH and acetylene related processes were assessed along the wings in the same manner as was done for the centerline of the ethylene/air flame and the results of that analysis can be seen in Figure 8. A distinct difference can be observed now wherein these processes are no longer close to being equal, but rather one is dominating the other. Along the wings, surface growth through the HACA mechanism dominates over the contribution that PAH related processes have on soot mass growth. 


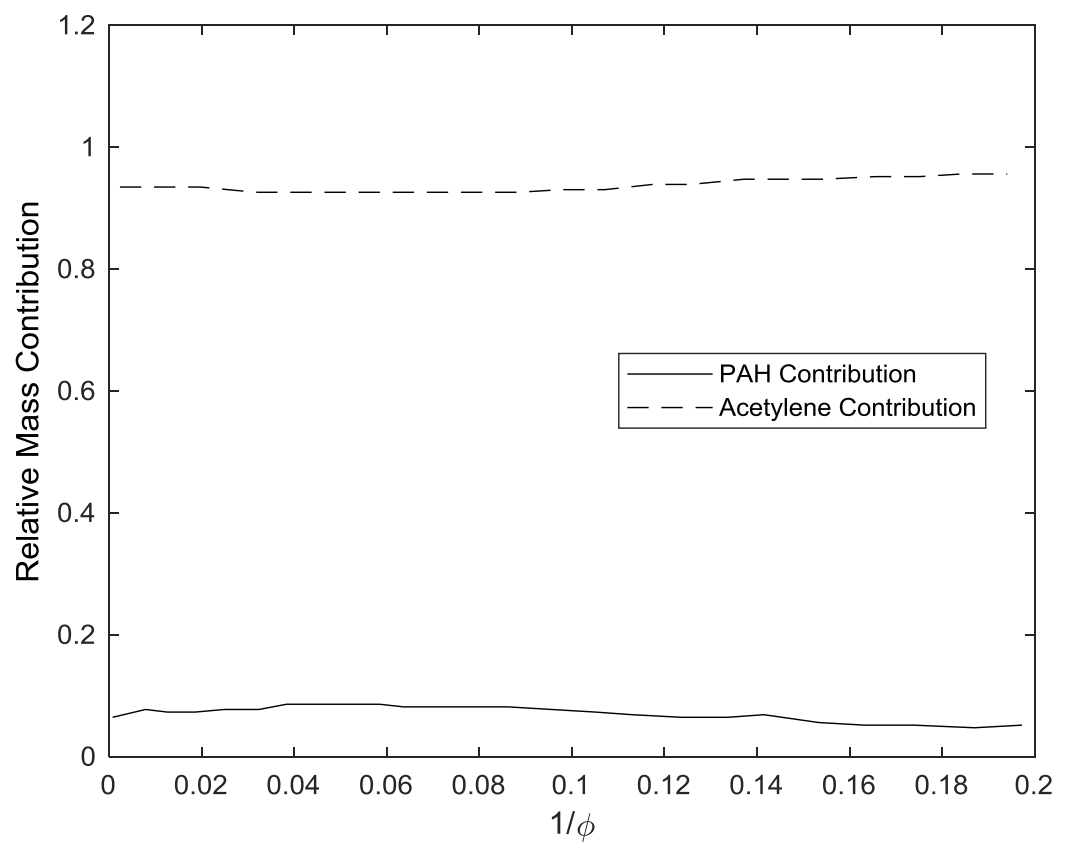

Figure 8: Relative mass contribution for Arana et al. [57] ethylene partially premixed flame along the wings for different equivalence ratios. Reproduced from Chernov et al. [10].

The importance of the findings in the work of Chernov et al. [10] is with regards to how the soot mass growth mechanisms affected each region of the flame and the significance of their contributions to soot formation. In the previous numerical study discussed [11], the finding that specific species concentrations can significantly influence the formation of soot was highlighted. Chernov et al. [10] builds on the characterization of the centerline behaviour as it presents how different soot mass growth mechanisms influence the formation of soot. One of the objectives of the present thesis is to be able to model aliphatic contributions to soot mass growth in such a way that the peak soot volume fraction along the wings remains unchanged while the centerline is improved. In order to do so, the behaviour of the wings must also be understood in a similar fashion to the behaviour of the centerline. Only by understanding which processes are critical in the formation of soot in different regions of the flame can a model be correctly developed. As briefly discussed, surface reactivity is a tunable constant, albeit to a limited extent, and is 
significantly influential in HACA growth, which further stresses the need to understand what processes affect which regions.

\subsubsection{Application to Different Fuel Carbon Composition}

One of the recommendations in the work of Dworkin et al. [11] was that other fuels should be tested. Since the original inception of the Slavinskaya and Frank mechanism, modifications have been made to account for advances in the current literature. The kinetic mechanism was further improved upon in [12] by modifying the PAH gas phase growth to improve soot volume fraction predictions in methane flames. The modifications were tested on methane [29], ethylene, and ethane [59] coflow laminar diffusion flames using a surface reactivity of 0.15 . To ensure no degradation had occurred for $C_{2}$ fuels, the changes were also tested for a methane/oxygen and two ethane/oxygen premixed flames.

One of the major findings was that the modifications that were made caused significant changes in the methane flame whereas the other flames remained relatively close to the unmodified numerical results along the centerline. Furthermore, the $C_{2}$ fuels had increased soot volume fraction predictions along the wings above experimental values, which was attributed to the higher surface reactivity that was used in the work of Chernov et al. [12]. The wings of the methane flame did experience an improvement in the soot volume fraction distribution without overshooting the experimental values. The centerline had increased significantly more so than when compared to the wings. This finding was attributed to the improved PAH gas phase growth, which improved the prediction of pyrene concentrations within the methane flame [12]. Methane flames have lower concentrations of acetylene and thereby experience lower rates of 
surface growth as compared to $C_{2}$ fuels. This makes methane flames more dependant on the accuracy of the chemical kinetic mechanism as surface growth cannot compensate as much.

The work of Chernov et al. [12] provided insight into the behaviour of methane coflow laminar diffusion flames. The major findings that were highlighted before to be important for the present thesis are once again illustrated in Chernov et al. [12]. The mechanisms associated with different regions of the flame through which soot mass is generated explained the results that were seen. The improvement that was seen in the centerline was in agreement with inception rates being closely related to soot yield in $C_{1}$ fuels. Methane flames are more sensitive to the changes in the gas phase chemistry as surface reactivity cannot compensate for soot inception deficiencies as significantly as can be done for $C_{2}$ fuels due to lower acetylene concentrations throughout the flame. As previously mentioned, the present thesis aims to simulate both methane and ethylene flame with modifications to the soot inception pathways, which requires understanding of the different behaviours of $C_{1}$ and $C_{2}$ fuels. The discussed studies provided a roadmap as to what is expected when making modifications to the inception mechanism and which mechanisms and corresponding variables are critical in the formation of soot. 


\section{Chapter 4 - Aliphatic Model Development, Testing, and Results}

\subsection{Overview}

As previously mentioned, this body of work has produced two different models that involve aliphatics influencing the routes of soot inception in two different ways. What is important to note for the entirety of this chapter is that the aim of the simulations is the exact same for both models. Earlier, it was stated that in the current literature the centerline of coflow laminar diffusion flames tends to be underpredicted by current numerical models. Furthermore, it was stated that aliphatic species may be able to influence soot formation in a manner that has not yet been discovered. The overall goal of the simulations is with regards to the results of the iterative process. The first condition is that the solution that has been generated must have a peak soot volume fraction along the wings close to the value outputted by the original model. This condition exists because the wings are better characterized in the literature than the centerline. The second condition is that the centerline soot volume fraction has to increase closer towards the experimental values when compared to the original model results. The reason for the second condition is in order to observe an improved soot distribution. The real aim of the simulations was to improve the ratio of the soot volume fraction between the wings and the centerline closer towards experimental values by using a physically justifiable approach, which is achieved when the two aforementioned conditions are met. The overall aim of the thesis was to see if aliphatics were capable of influencing this objective.

\subsection{Collision Based Aliphatic Inception}

\subsubsection{Model Description}

From the work of Wang et al. [16] and other studies [17, 18, 19], corroborating evidence suggests that nascent soot can be rich in aliphatics and that soot mass can grow without the 
presence of gas phase hydrogen atoms. The importance of these observations is with regards to the HACA mechanism as it requires the removal of hydrogen from the surface of soot particles in order to function. If hydrogen atoms are not present, then the HACA mechanism is not active and acetylene molecules are not being deposited onto the surface of these soot particles. This evidence indicates that another mechanism must be responsible for this growth, possibly involving aliphatics as their presence is detected in these nascent soot particles. Prior to this thesis, CoFlame only contained a single mechanism that directly related aliphatic concentrations to soot mass growth, which was through the HACA mechanism with acetylene molecules. The participation of the other aliphatic species was limited only to the chemical kinetic mechanism wherein eventually some of the smaller species would grow into PAHs that would result in the inception of soot while most of the aliphatics would oxidize. The present thesis has proposed a mechanism that links gas phase aliphatics to the solid soot phase. This provides a testing ground for which to relate the presence of aliphatics to a better soot distribution.

For the new mechanism, it is assumed that aliphatic molecules collide together and under the right conditions may stick together. These conditions are accounted for in the collision efficiency that is prescribed in the CoFlame code. This mechanism was developed to function in a similar manner to PAH dimerization. The primary difference between this mechanism and PAH dimerization lies in the specific molecules that are colliding and contributing to soot inception. PAH dimerization involves large PAHs participating in the inception of soot, such as BAPYR $\left(\mathrm{C}_{20} \mathrm{H}_{12}\right)$ whereas this mechanism uses specific aliphatic species for the same means. A value is given to the collision efficiency to indicate that only $X$ in every $Y$ collisions will result in effective sticking. For example, if the collision efficiency is set 0.01 , this indicates that 1 in every 100 
collisions will result in sticking, and the other 99 will result in a rebound. Once the molecules stick together, they are treated as an incipient soot particle. This in effect means that this mechanism is contributing to the inception of soot, in addition to traditional PAH routes, which are still included in the model. Furthermore, the collision rates are highly dependent on molecular dynamics. Each of the aliphatic molecules considered has an effective radius and a concentration. The size and concentration inherently have an influence on the soot mass growth as the number of collisions increases with an increase in either one of those two parameters. It is important to note that the purpose of this mechanism is to simply link the aliphatics in the gas phase to the incipient solid soot phase, so as to assess their potential impact on soot mass growth and spatial distribution. A visual illustration of this mechanism can be seen in Figure 9.<smiles>CC(C)(C)C1C#CCC#C1</smiles><smiles>CC#CC</smiles>

\section{NASCENT SOOT PARTICLE}

Figure 9: Visual representation of the collision based aliphatic inception model moving two aliphatic species, which in this case would be $\mathrm{C}_{4} \mathrm{H}_{6}$, from the gas phase to the solid soot phase.

The rate of soot inception is given by:

$$
\frac{\partial N}{\partial t}=\beta \sqrt{\frac{8 \pi k_{B} T}{\mu_{A B}}}\left(r_{A}+r_{B}\right)^{2} A_{V}^{2}[A][B]
$$

Where $[A]$ and $[B]$ are the concentrations of the two colliding aliphatic species [46]. 
The present study implements a 94-species chemical kinetic mechanism, which models ethylene or methane combustion and PAH growth [23]. Some of the aliphatic species have been filtered out of use in the inception model based on their concentrations and carbon mass. Extremely low concentrations with low carbon mass such as $\mathrm{CH}_{2}$ have been removed from the proposed mechanism. From this analysis it was determined that only 6 species of aliphatics are of particular interest in the present study. Those species are: $\mathrm{CH}_{4}$ (methane), $\mathrm{C}_{2} \mathrm{H}_{2}$ (acetylene), $\mathrm{C}_{2} \mathrm{H}_{6}$ (ethane), $\mathrm{C}_{3} \mathrm{H}_{6}$ (propene), $\mathrm{C}_{4} \mathrm{H}_{6}$ (butyne), and lastly $\mathrm{C}_{3} \mathrm{H}_{8}$ (propane).

While the present model does not represent the complete physics behind the problem, it can be used to determine the potential for mass transfer from the aliphatic gas phase to the soot solid phase. This strategy allows for analysis to be carried out, such that relationships can be made between the aliphatic species and soot mass growth. Adjusting the collision efficiency of aliphatics, and further adjustment to the soot surface reactivity parameter, as was done in [11], are key components to this exercise. Increasing the collision efficiency leads to more soot mass growth whereas decreasing it has the opposite effect. Through this adjustment, it is hypothesized that the centerline soot volume fraction can be modified to better predict experimental values along the centerline of laminar diffusion flames, and in turn better understand mechanisms of soot particle formation.

Another key factor in determining the overall soot volume fraction prediction of the numerical model is the soot surface reactivity. The surface reactivity is a value that ranges from 0 to 1 and is representative of the portion of a soot particle's surface area that is available for chemical reaction. CoFlame allows for this surface reactivity to be modelled as either a constant or a function of temperature history. This parameter is a factor in determining HACA growth and 
oxidation. Decreasing the surface reactivity normally leads to a decrease in the soot volume fraction as the contribution of the HACA mechanism to surface growth decreases. The opposite is normally true when the surface reactivity is increased. Further adjustment of the surface reactivity, beyond what is already provided in the literature $[11,53]$ becomes relevant when a new growth mechanism is introduced into the numerical model. Since the surface reactivity parameter was tuned with a previous model, introducing a new mechanism may no longer produce realistic quantities of soot with the same value for surface reactivity. Thus, surface reactivity must also be balanced in tandem with the collision efficiency in order to obtain meaningful results for this research.

\subsubsection{Results and Discussion}

Once the model was fully integrated into the CoFlame code, the first step in the simulation procedure was in determining whether or not the collision efficiency for the aliphatics should be represented as either a constant value or a functional form. A functional form may be more representative of the actual physics behind the problem as collision efficiency is dependant on many different variables within a molecular dynamics framework, such as mass and molecular symmetry [20]. However, it was decided that a constant value would be used. Many of the collisions that occur are between different aliphatic species and therefore it is not likely that a single collision efficiency could prescribe all those collisions, but it can act as a first step towards the generation of a fundamental model. Similarly, the surface reactivity can be modelled as a function but was kept a constant value for the same reason that collision efficiency was kept constant. Furthermore, the functional form for surface reactivity was derived from previous 
numerical results [54], which could potentially cause some issues in the model when introducing a new inception mechanism that changes the output results.

After it was decided that a constant value for the collision efficiency and surface reactivity would be used, the iterative process could begin. As discussed in the previous chapters, both of these terms influence the formation of soot along the wings and the centerline differently. Collision efficiency is directly responsible for the rate of soot inception, which is of great importance for the inception dominated centerline. The surface reactivity affects both the centerline and the wings, but the wings experience significantly higher rates of surface growth due to higher concentrations of acetylene. Table 3 is the summary of the iterations that were done for both the surface reactivity and collision efficiency in order to achieve the simulation goals outlined in the overview. Moreover, the NSII flame was the first to be tuned because of its prevalence in the literature and better characterization in contrast to the methane flame.

Table 3: Collision Based Aliphatic Inception Iterations Results for the NSII Flame

\begin{tabular}{|c|c|c|c|}
\hline \multirow{3}{*}{ Surface Reactivity $(\alpha)$} & Collision Efficiency & \multicolumn{2}{|c|}{ Peak Soot Volume Fraction (ppm) } \\
\cline { 2 - 4 } & $(\beta)$ & Wings & Centerline \\
\hline \multirow{5}{*}{0.1} & $1 \mathrm{e}-4$ & 275 & 2.97 \\
\cline { 2 - 4 } & $1 \mathrm{e}-6$ & 157 & 5.67 \\
\cline { 2 - 4 } & $1 \mathrm{e}-8$ & 36.50 & 4.86 \\
\cline { 2 - 4 } & $1 \mathrm{e}-10$ & 7.23 & 2.79 \\
\cline { 2 - 4 } & $1 \mathrm{e}-12$ & 3.73 & 2.24 \\
\hline \multirow{5}{*}{0.2} & $1 \mathrm{e}-8$ & 54.20 & 3.33 \\
\cline { 2 - 4 } & $1 \mathrm{e}-10$ & 13.10 & 3.10 \\
\cline { 2 - 4 } & $1 \mathrm{e}-12$ & 5.30 & 2.47 \\
\cline { 2 - 4 } & $1 \mathrm{e}-14$ & 5.05 & 2.45 \\
\hline \multirow{5}{*}{0.3} & $1 \mathrm{e}-16$ & 5.04 & 2.45 \\
\hline & $1 \mathrm{e}-10$ & 19.50 & 4.12 \\
\hline & $1 \mathrm{e}-11$ & 9.83 & 2.84 \\
\cline { 2 - 4 } & $1 \mathrm{e}-12$ & 7.25 & 2.63 \\
\cline { 2 - 4 } & $1 \mathrm{e}-13$ & 6.88 & 2.60 \\
\hline
\end{tabular}


Without the aliphatic inception model, a surface reactivity of 0.85 was used in CoFlame to achieve numerical predictions close to the experimental values. Those results showed a peak soot volume fraction along the wings of $9.72 \mathrm{ppm}$ and $2.42 \mathrm{ppm}$ along the centerline. This information acted as the baseline for which to compare the results of the simulations for the aliphatic inception model. As a starting point, it was decided that the surface reactivity would be lowered from 0.85 from the original model to 0.1 for the new inception model. This was done to reduce the influence of surface growth without completely eliminating it in order to be able to better understand how the collision efficiency for aliphatics influenced the formation of soot. Furthermore, the collision efficiency that was used for the aliphatics was the same value as for the PAHs, which was 1e-4. The results of that simulation, as shown in Table 3, generated large amounts of soot along the wings compared to the original model. The centerline did not experience as intense of an increase in soot formation. This did not only indicate that the selected collision efficiency was too high, but also that the selected aliphatic species were more concentrated along the wings. The next step was to test a range of collision efficiencies and it was decided that the range would be between $1 \mathrm{e}-4$ and 1e-12. Below a collision efficiency of 1e8 , the prediction along the wings decreased to a point where the original model had a higher prediction. This decrease occurred for a collision efficiency of 1e-10, however, the centerline had simultaneously increased relative to the original model results.

Veshkini et al. [60] determined that the optimal value for the surface reactivity for the NSIl flame was 0.45 . Thus, it was decided to increase the surface reactivity for the following simulations. It may have been possible to achieve the desired simulation goal detailed in the 
overview using a surface reactivity of 0.1 as multiple solutions may exist. However, it was of interest to observe the influence that surface reactivity had when applied in tandem with the new inception model. Thus, the iterative process continued by increasing the surface reactivity to 0.2 as seen in Table 3. A collision efficiency of $1 \mathrm{e}-10$ and a surface reactivity of 0.1 resulted in values closer to the original model results and was thus used as the basis for the generation of the collision efficiency range for the next tested surface reactivity. The same procedure of using the previous results to select a range of values for the collision efficiencies for the subsequent surface reactivity was repeated once more for a surface reactivity of 0.3 .

Figure 10 shows the predicted soot volume fraction along the pathline of maximum soot of the NSII flame as a function of height above the burner for a collision efficiency of $1 \mathrm{e}-11$ and a surface reactivity of 0.3 . The data is compared to the original model without aliphatic inception and to experimental results. It can be seen from Figure 10 that the predictive capability along the wings of the flame are generally unaffected by the addition of aliphatic inception. Furthermore, only collision efficiencies less than 1e-10 can lead to reasonable results with a surface reactivity of 0.3 as using any higher collision efficiencies will result in the over prediction of the soot distributions. Adding aliphatic inception, however, shifts the location of soot formation to higher heights above the burner, away from the experimental peak. According to the new numerical results, the way the peak shifts, indicates that soot formation as well as oxidation is occurring higher above the burner. 


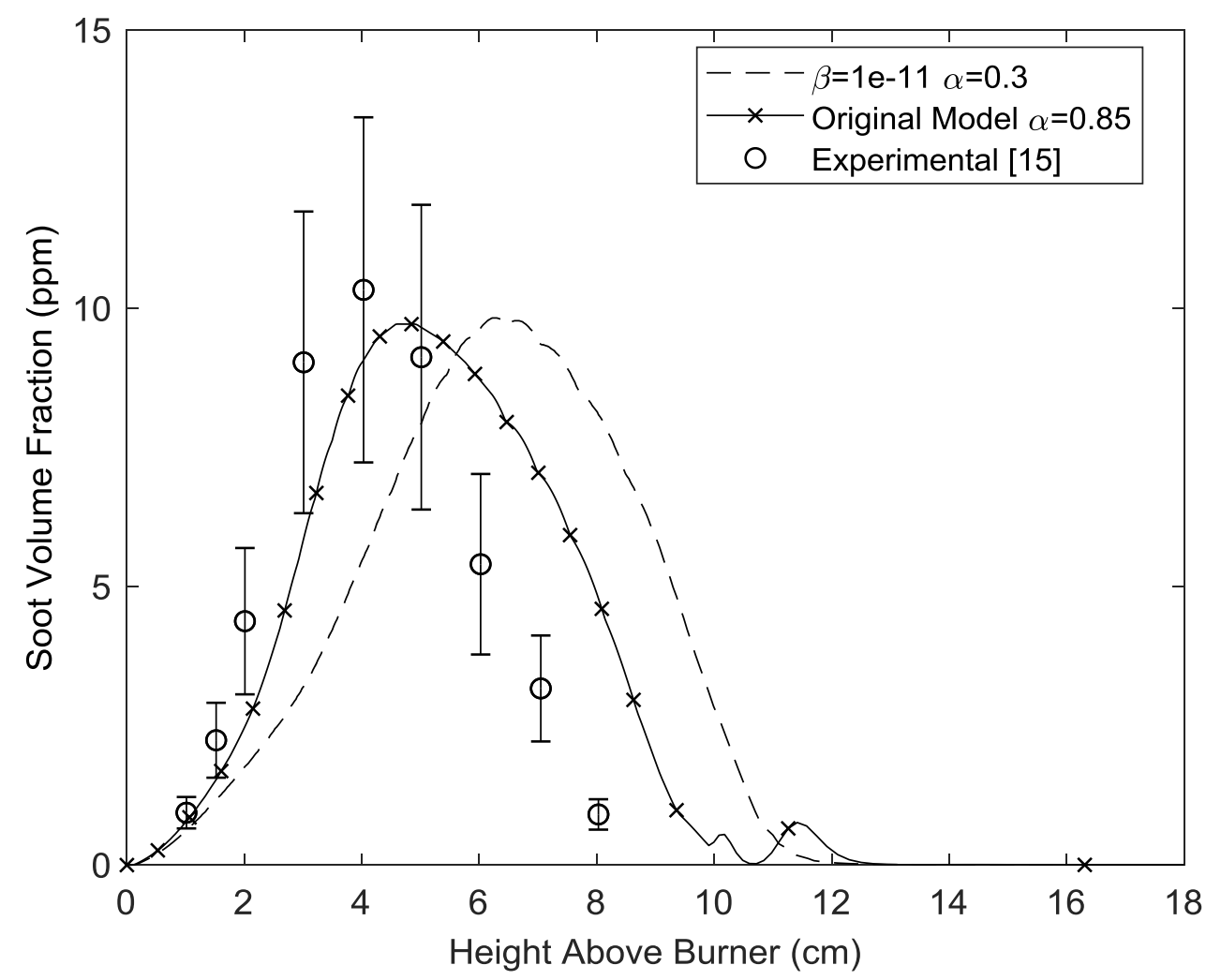

Figure 10 Predicted soot volume fraction as a function of height above the burner for a collision efficiency of 1e-11 with constant surface reactivity of 0.3 along the wings of the NSII flame. The results of a model without aliphatic inception and a surface reactivity of 0.85 is shown alongside experimental results from [15].

Figure 11 shows the predicted soot volume fraction along the centerline of the NSII flame as a function of height above the burner for the same collision efficiency and surface reactivity. Here too the data is compared to the original model without aliphatic inception and to experimental results. Again, only collision efficiencies less than $1 \mathrm{e}-10$ lead to physically realistic results. Otherwise, implementing aliphatic based inception results in only a modest increase of the soot volume fraction along the flame centerline. Figures 10 and 11 illustrate that when $\alpha$ is set to 0.3 and $B$ is set to $1 \mathrm{e}-11$, there is an increase in the soot volume fraction along the centerline while the prediction along the wings remains close to the experimental peaks. The same upward peak shifting phenomenon can be observed along the centerline in Figure 11 . The results of varying the collision efficiency and surface reactivity showed that for the NSII flame, $\alpha$ 
can be lowered to a more physically realistic [60] value in order to correctly predict the peak soot volume fraction along the wings. In order to obtain results close to the experimental peaks for the NSII flame using CoFlame without the new inception model, $\alpha$ had to be set to 0.85 .

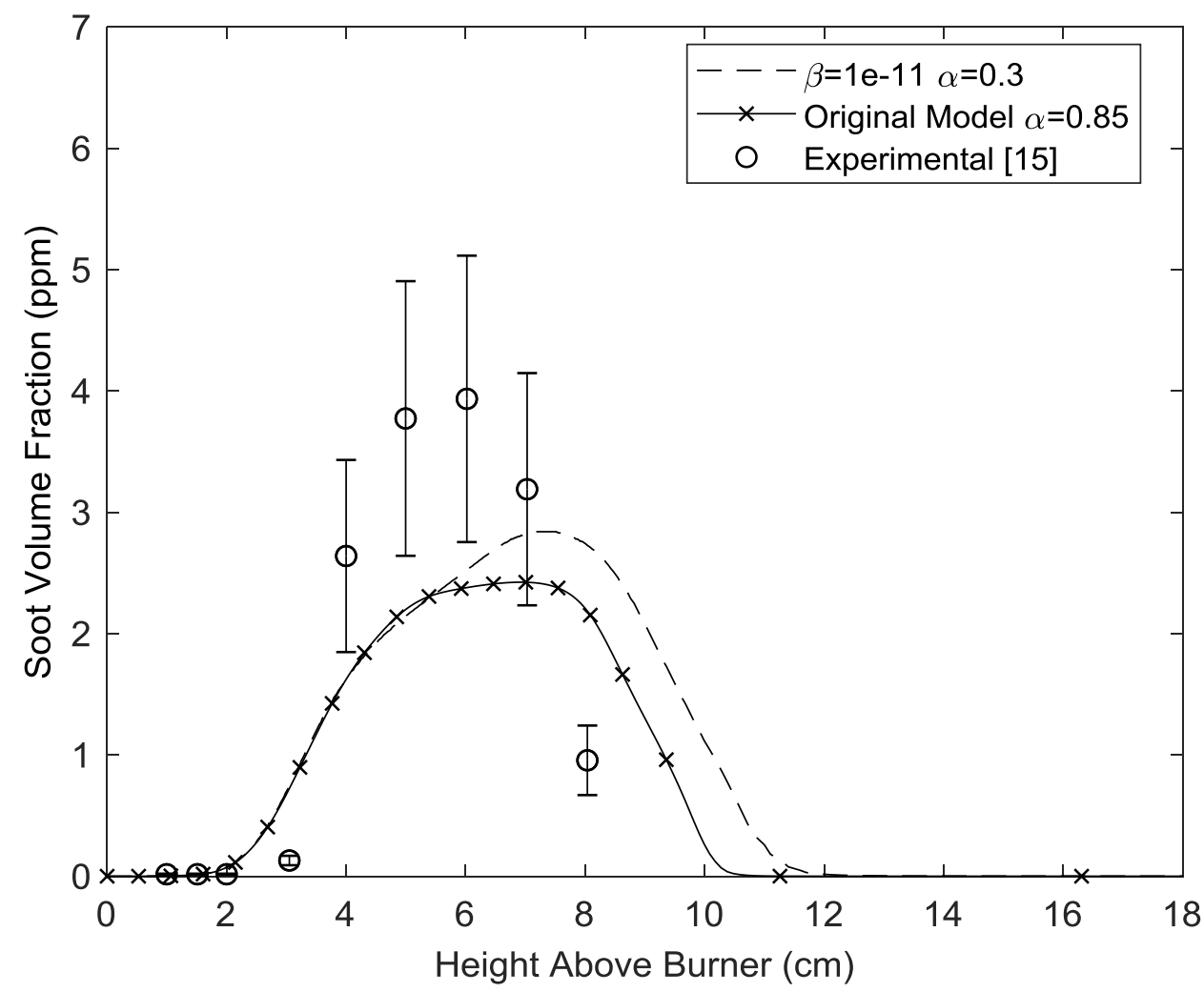

Figure 11: Predicted soot volume fraction as a function of height above the burner for a collision efficiency of $1 \mathrm{e}-11$ with constant surface reactivity of 0.3 along the centerline of the NSII flame. The results of a model without aliphatic inception and a surface reactivity of 0.85 is shown alongside experimental results from [15]

Similarly, the predicted soot volume fraction of the methane flame as a function of height above the burner for varying collision efficiencies and surface reactivity compared to the original model without aliphatic inception and experimental results is shown in Figures 12 and 13 . The results along the wings of the methane flame are shown in Figure 12 while Figure 13 shows the data along the centerline. Using the same values for $\alpha$ and $B$ as for the NSII flame that achieved the overall simulation goal, 0.3 and $1 \mathrm{e}-11$ respectively, resulted in the under prediction of the soot volume fraction in both the centerline and wings. In ethylene flames, the surface reactivity 
can compensate for deficiencies in the chemical kinetic mechanism [12]. However, since methane flames are less influenced by HACA growth, the deficiencies in the reaction scheme remain prevalent. The latest version of CoFlame has added reactions specifically for methane flames as detailed by Chernov et al. [12]. Although there is an improvement in the model's predictive capability, increasing soot concentrations, even when the surface reactivity is set to its theoretical limit of unity, both regions of the flame remain under predicted. When collision based aliphatic inception is applied with the same $B$ of $1 \mathrm{e}-11$ as before and $\alpha$ is increased to 1 , there is an increase in the soot volume fraction along both the centerline and wings. Once again, the same peak shifting phenomenon can be observed. The collision based aliphatic inception model is able to improve the comparisons to experimental data, but not rectify all remaining inaccuracies in the methane flame.

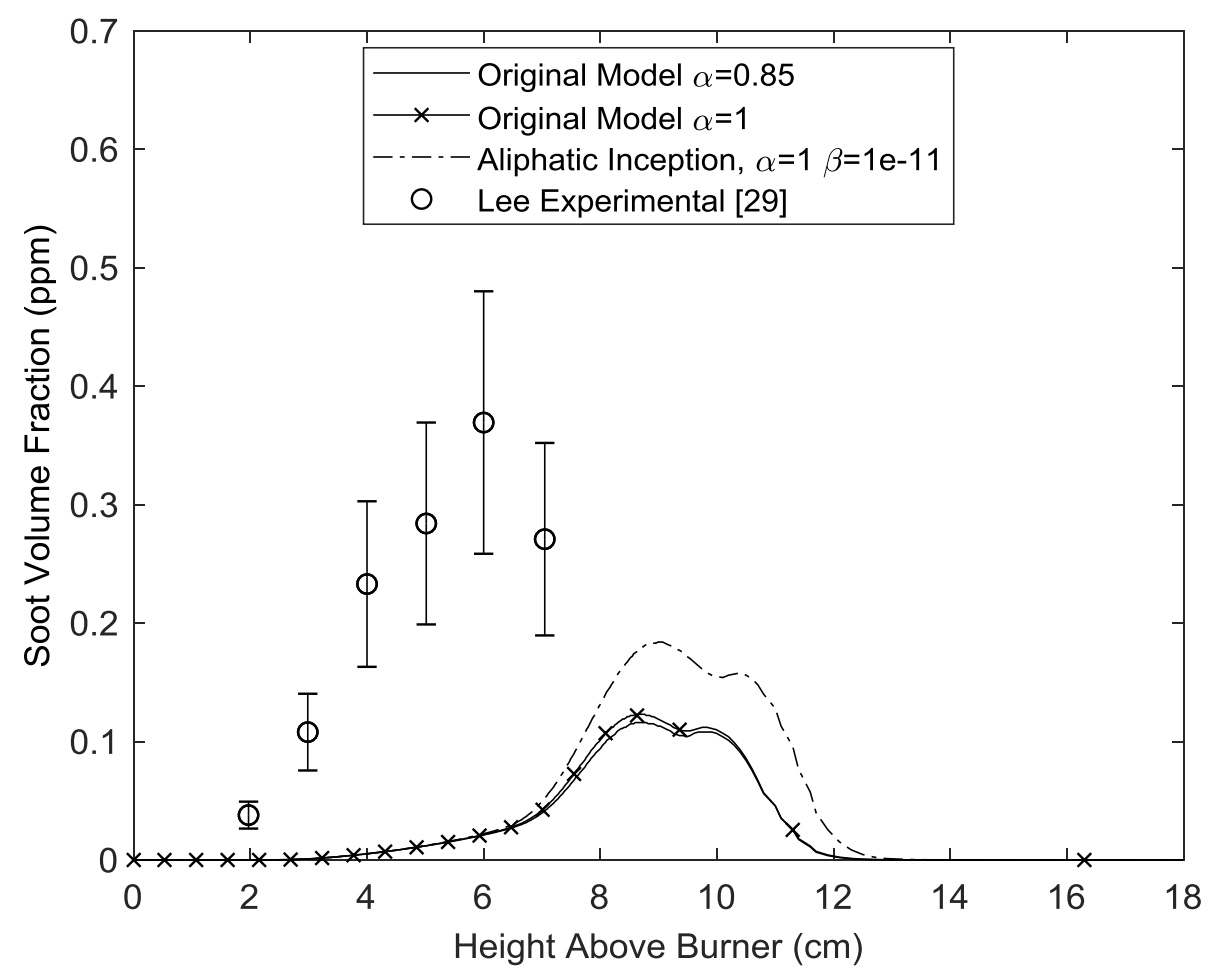

Figure 12: Predicted soot volume fraction as a function of height above the burner for a model without aliphatic inception using $\alpha$ of 0.85 and 1.0 along the wings of the methane flame. The aliphatic inception model is shown using the same $\beta$ as for the NSII, 1e-11. The experimental work of [29] is plotted. 


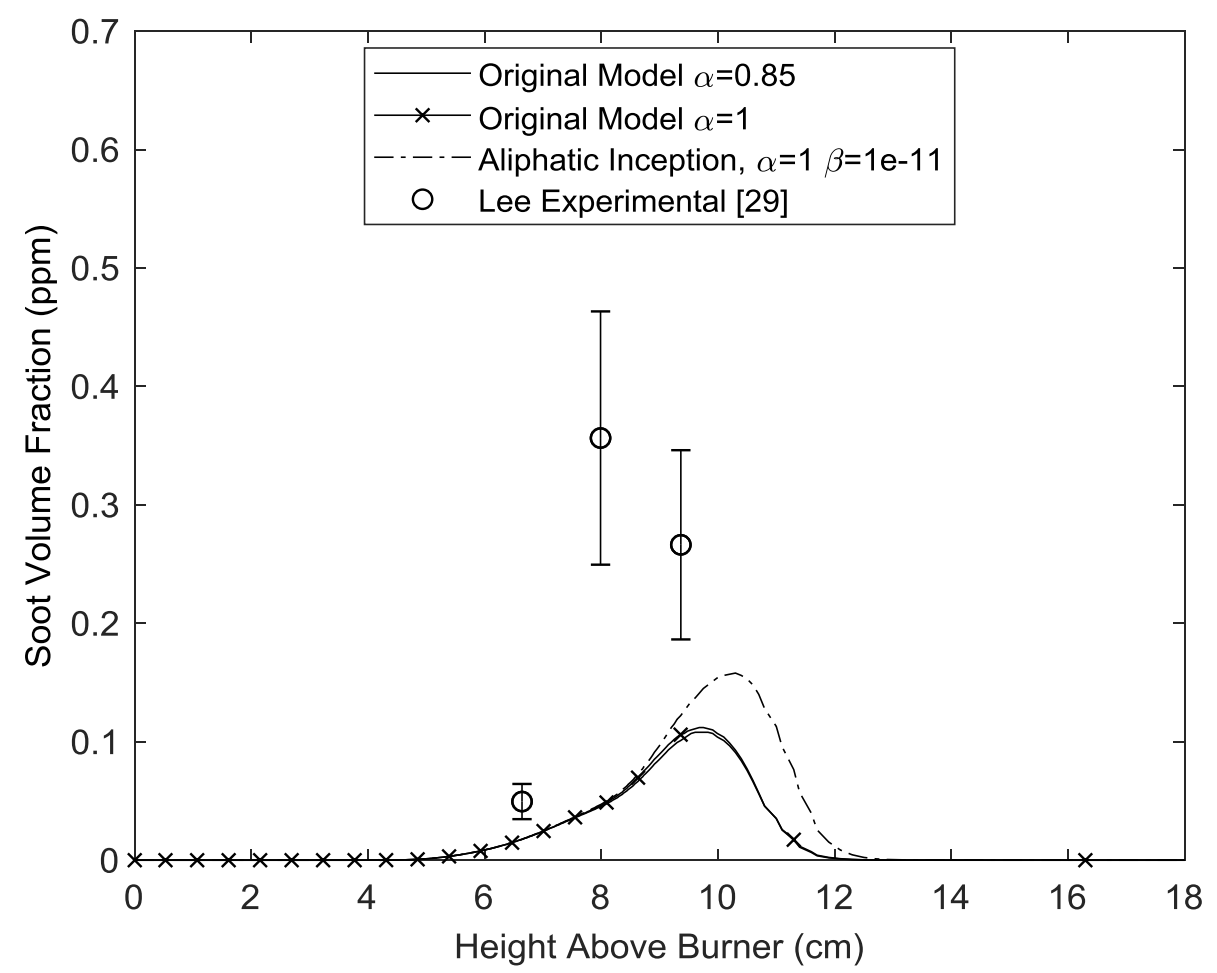

Figure 13: Predicted soot volume fraction as a function of height above the burner for a model without aliphatic inception using $\alpha$ of 0.85 and 1.0 along the centerline of the methane flame. The aliphatic inception model is shown using the same $\beta$ as for the NSII, 1e-11. The experimental work of [29] is plotted.

Theoretically, further tuning could have been carried out to achieve the best results that could bring the numerical model's prediction as close to the experimental values as possible for both of the flames. However, fundamental issues are prevalent in the model with regards to some of the underlying physics. As it was stated earlier, the intention of this model was to not to be completely fundamental in nature but more so to establish that a relationship might exist with aliphatic species and how they might influence the centerline soot volume fraction. Thus, more literature was surveyed to improve the model and the results of that survey will be discussed in the following section.

\subsection{Collision Based Aliphatic Stabilization}

\subsubsection{Model Description}

Evidence from the work of Chung and Violi [21] suggested that soot precursor species with aliphatic chains were more likely to result in dimerization than when compared to the same 
species without these aliphatic chains. Furthermore, the species of aliphatics that stabilized these dimerization events contained 3 carbons and higher and the resulting combined molecules could further lead to growth into aggregate structures [20]. The results also suggested that the rate of nucleation was faster because the molecules were able to better accommodate the impact energy into additional internal vibrational modes [21]. Taking into consideration this evidence along with the evidence that was provided in the previous model description lead to the generation of a new aliphatic inception model.

For this new mechanism, modifications were made to the previous model that significantly changed how the physics was being modelled. Rather than having the collisions between two aliphatic species resulting in the inception of soot, now the model has been changed such that PAH based inception is influenced by specific aliphatic species. These aliphatics are simply there as stabilizing molecules for the collisions that occur between large PAHs that result in the inception of soot. As previously mentioned, these large PAHs are BAPYR, BAPYRS, and BGHIF. This collision stabilization is achieved by generating a functional form for the collision efficiency that prescribes the rate of soot inception to be dependant on the concentrations of 3 carbon and higher aliphatic species. Within the 94-species chemical kinetic mechanism, the aliphatics that contain 3 carbons and higher are $\mathrm{C}_{3} \mathrm{H}_{6}$ (propene), $\mathrm{C}_{4} \mathrm{H}_{6}$ (butyne), and $\mathrm{C}_{3} \mathrm{H}_{8}$ (propane) and were thus used for this new mechanism. Essentially, when a successful collision occurs between these large PAHs, both the PAHs as well as the selected aliphatic species are removed from the gas phase and moved into the solid phase. The handling of this is done in a 1 to 1 to 1 ratio wherein 1 of each of the collided PAHs and 1 of the 3 carbon aliphatic species is moved. A visual illustration of this can be seen below in Figure 14. The rate equation is given to 
be the same as for the previous model, with the addition of the aliphatics to the reaction sequence.
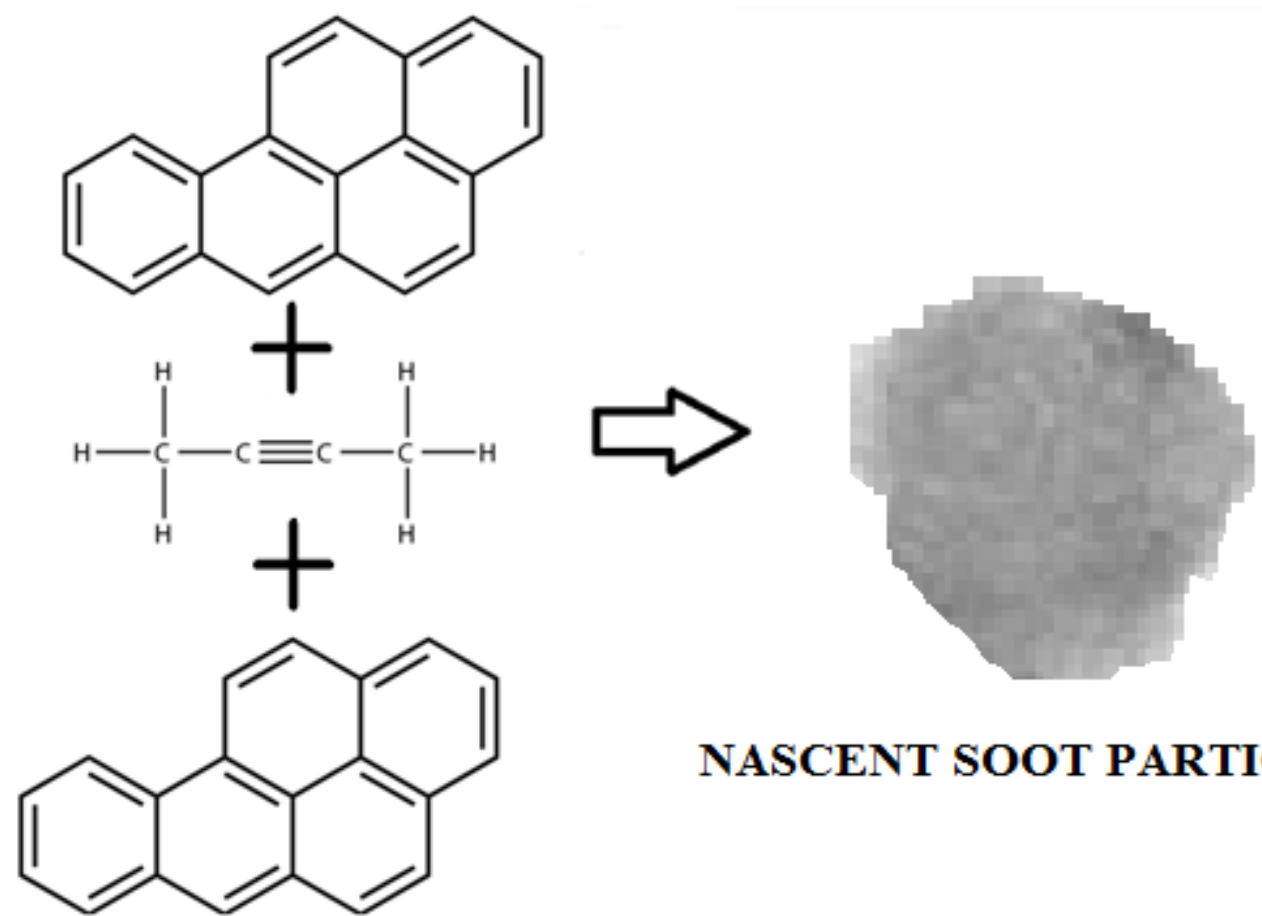

NASCENT SOOT PARTICLE

Figure 14: Visual representation of the collision based aliphatic stabilization model moving two aliphatic species, which in this case would be $\mathrm{C}_{4} \mathrm{H}_{6}$, from the gas phase to the solid soot phase.

In contrast to the previous model, this model treats PAH and aliphatic contributions as a single route towards the inception of soot whereas the previous model treated them separately. This change would address one of the major flaws in the previous model wherein the physics are more justifiable. The collision between aliphatics is not likely to result in the inception of soot, however, it is more physically justifiable that these species could influence the rate at which larger PAHs become soot based on evidence from the literature provided.

The generation of the function prescribing collision efficiency as being dependant on aliphatic species concentration requires an iterative scheme that is of a similar nature to the previous model with some slight changes in the methodology. Generating a function involves 
proposing the order of the function and the constants in front of the independent variables need to be solved iteratively in order to achieve the simulation goal mentioned in the overview. Compared to the methodology that was used for the inception model, the iterative scheme is essentially the same wherein both surface reactivity and collision efficiency are computed iteratively. However, the major difference in this case is the iterative computing of the collision efficiency is no longer a constant value, but rather of a functional form, which carries different characteristics and considerations when developing the iterative scheme.

\subsubsection{Results and Discussion}

The first step in the generation of the function was in the determination of how each of the aliphatic species would be represented. For simplicity, it was decided that rather than having individual terms for the aliphatics species concentrations, which there are three of, that the function would only consider a superimposed aliphatic concentration field. Numerically this would be expressed by the following equation:

$$
f\left(x_{1}, x_{2}, x_{3}\right)=C_{1}\left[x_{1}+x_{2}+x_{3}\right]
$$

Where $x_{i}$ is the concentration of the $i^{\text {th }}$ species.

The aliphatic field would simply be a summation of the concentration of each of the 3 carbons and higher aliphatics present in the steady state solution. One of the critical issues that would arise with treating the three species independently from each other within the function would be in the iterative computation of the function constants. Numerically this would be expressed by the following equation:

$$
f\left(x_{1}, x_{2}, x_{3}\right)=C_{1}\left[x_{1}\right]+C_{2}\left[x_{2}\right]+C_{3}\left[x_{3}\right]
$$


If there are 3 terms that need to be iteratively computed rather than 1 , the number of iterations required is significantly increased as the number of possible solutions is greater than when compared to a single constant. Inherently, by making this assumption it also assumes that the aliphatic species will be contributing equally to stabilization, which is physically unlikely to occur as the molecules are of different geometries. However, as a first step in the generation of the function, the superimposed field assumption was made and was justifiable as the selected aliphatics are of relatively similar geometries.

Using the original numerical model and a surface reactivity of 0.85 , the aliphatic concentrations of $\mathrm{C}_{3} \mathrm{H}_{6}, \mathrm{C}_{4} \mathrm{H}_{6}$, and $\mathrm{C}_{3} \mathrm{H}_{8}$ were computed and plotted in Figure 15 for the NSII flame. The NSII flame was selected to be the first flame for iterations because of the same reasons that were outlined in the previous model. The resulting superimposed concentration field for the summation of the aliphatics can be seen in Figure 15 as well. It should be noted that the highest superimposed concentrations occur along the wings and close to the centerline. The absolute highest superimposed concentration occurs along the wings close to the edge of the burner. 


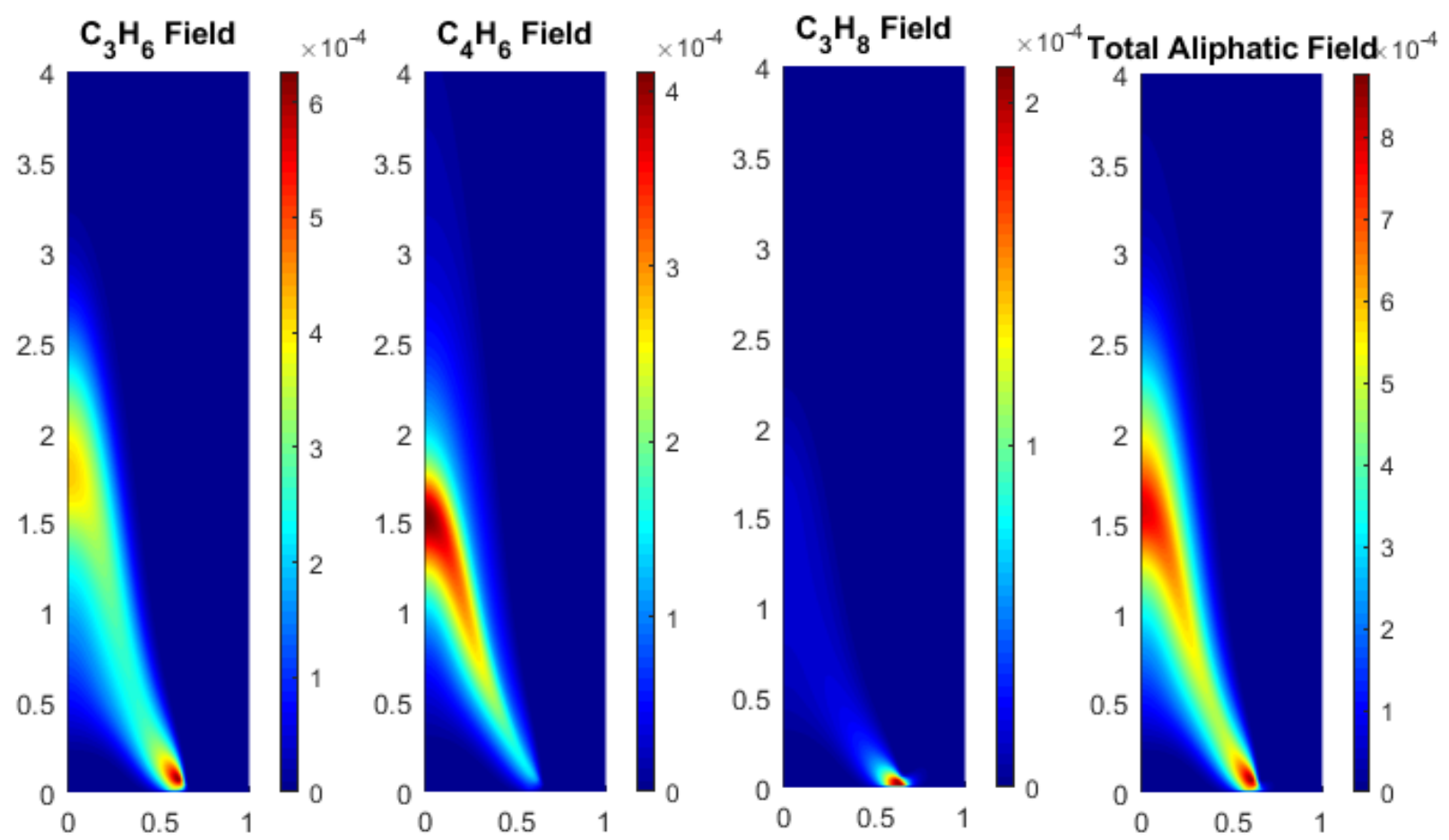

Figure 15: Computed aliphatic species concentrations using the original model and a surface reactivity of 0.85 for the NSII flame. The superimposed aliphatic field represents the summation of the aliphatic species presented. The results are expressed in ppm.

The next step had been in determining the order of the function. It was decided that a first order linear function would be generated because of how the collision efficiency was going to be prescribed. Since the dimerization of PAHs was going to be dependant on aliphatic species concentrations in a 1 to 1 to 1 ratio, this meant that the collision efficiency would be directly proportional to the species concentrations. Therefore, it followed that the only viable function would be a linear one as it indicates direct proportionality, unlike a quadratic function that would be proportional to the square of species concentrations. The linear function had the following general form:

$$
f\left(x_{1}, x_{2}, x_{3}\right)=C_{1}\left[x_{1}+x_{2}+x_{3}\right]+C
$$

The constant term, $\mathrm{C}$, was given a value of 0 because the new model assumes that each effective collision between large PAHs also moves aliphatics from the gas phase to the solid phase. 
The iterative computation of the constant in front of the aliphatic species concentration term in the linear function, $C_{1}$, was handled by using previous numerical results for the steady state aliphatic concentrations. The new model removes aliphatic species from the gas phase when soot inception occurs. Thus, using the previous aliphatic concentration field to conduct the iterative computations will result in a different collision efficiency field once the new model reaches steady state conditions. However, an arbitrary starting point must be selected from which to begin the iterations. Therefore, it was decided that the initial peak collision efficiency at any given point in the field should be no greater than the constant value for the PAH collision efficiency, which was $1 \mathrm{e}-4$. In making this assumption, the functional constant can be computed to provide a starting point for the iterations. It follows that:

$$
C_{1}=\frac{1 e-4}{\left[\operatorname{MAX}\left[x_{1}+x_{2}+x_{3}\right]\right]}=\frac{1 e-4}{8.7786 e-4}=0.1139
$$

The equation then becomes:

$$
f\left(x_{1}, x_{2}, x_{3}\right)=0.1139\left[x_{1}+x_{2}+x_{3}\right]
$$

Figure 16 shows the concentration fields for the various large PAHs that participate in the inception of soot as well as the computed collision efficiency field for the proposed linear function. One area of concern is with regards to the mismatch in the peak concentration of PAHs in relation to the selected aliphatic species concentrations. Along the wings, the concentration of these species appears to be relatively close to each other at the edge of the burner. However, the peak aliphatic concentrations along the centerline occur around $1.5 \mathrm{~cm}$ above the burner whereas the peak PAH concentrations occur in between 2 to $3 \mathrm{~cm}$ above the burner. Since $\mathrm{C}_{1}$ was 
derived using the peak summed aliphatic concentration, which occurs along the wings, the centerline will be affect residually because the derivation was inherently focused on the wings while there is a mismatch between the concentration of the aliphatics and PAHs.
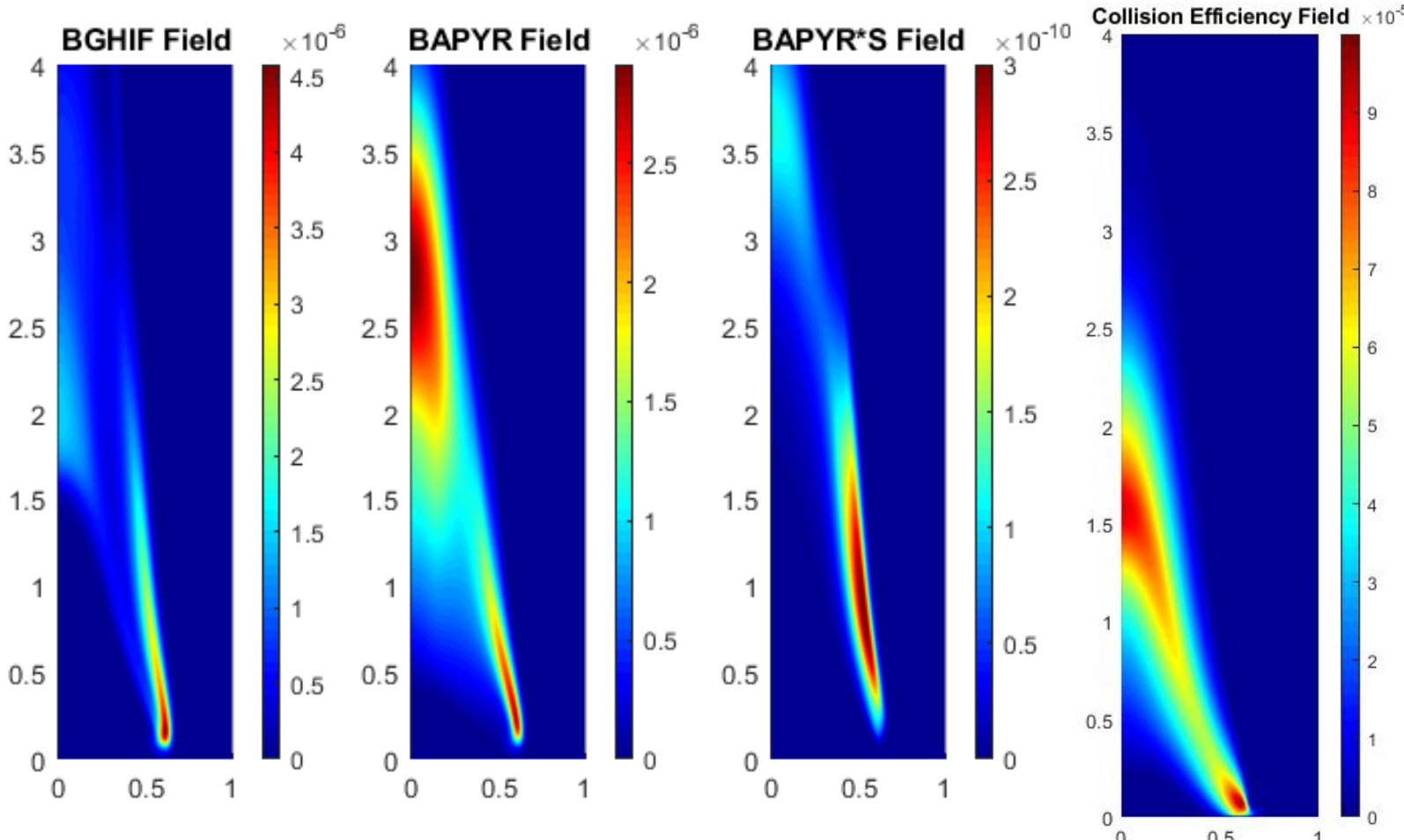

Figure 16: Computed PAH species concentrations using the original mechanism and a surface reactivity of 0.85 for the NSII flame. The results are expressed in ppm. Additionally, the computed collision efficiency of the form $f(x 1, x 2, x 3)=0.1139[x 1+x 2+x 3]$ is plotted.

The last consideration that was made before beginning the simulations was that the concentration of aliphatic species was derived from the original model with a surface reactivity of 0.85 . Thus, the surface reactivity was kept constant for the first set of simulations. This was done to minimize the effects that the surface reactivity would have on the soot formation. Figure 17 shows the numerical results for the soot volume fraction along both the centerline and wings using the proposed linear function to prescribe collision efficiency for the NSII flame plotted alongside the original model predictions and experimental results. 


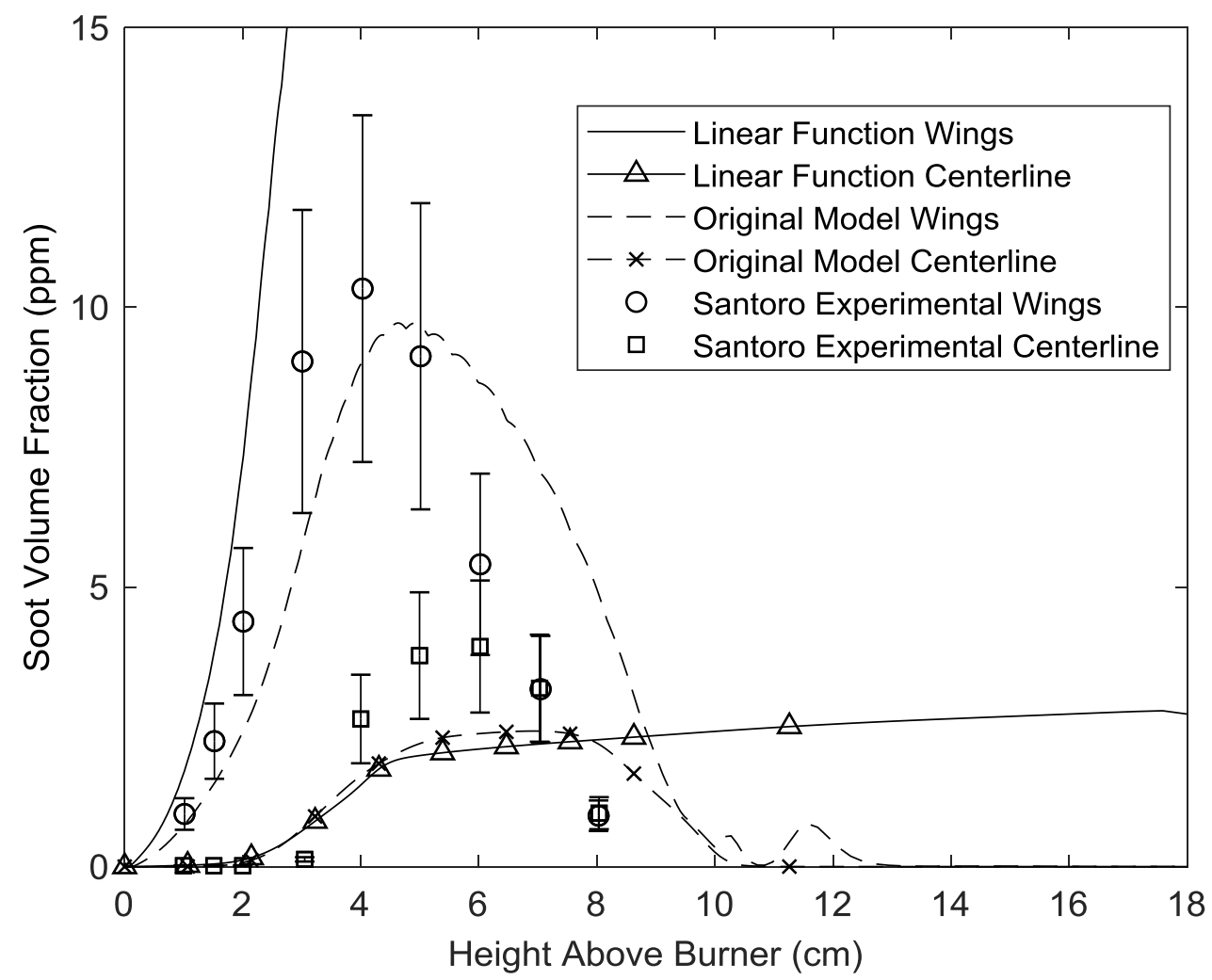

Figure 17: Predicted soot volume fraction as a function of height above the burner for the stabilization model with a collision efficiency prescribed by $f\left(x_{1}, x_{2}, x_{3}\right)=0.1139\left[x_{1}+x_{2}+x_{3}\right]$ along the wings and centerline of the NSII flame. The results of the original model are shown alongside experimental results from [15]. The surface reactivity was constant between the two models and had a value of 0.85 . The collision efficiency for the original model was a constant value of $1 \mathrm{e}-4$.

The amount of soot formed was significantly overpredicted along both the wings and the centerline, which had peak values of $24.39 \mathrm{ppm}$ and $2.79 \mathrm{ppm}$, respectively. The centerline did not overpredict the peak soot volume fraction, but it did not correctly capture the trend as soot oxidation was occurring at greater heights above the burner. Therefore, the next step in the procedure was to multiply the constant $C_{1}$ with different orders of magnitude. From Figure 17 , it can be seen that assuming that the collision efficiency field could not be greater than $1 \mathrm{e}-4$ did not achieve the simulation goals. However, it did provide a starting point from which to lower the collision efficiency field. Therefore, it was decided that $C_{1}$ would be multiplied by different 
orders of magnitude to observe the resulting effects on the soot volume fraction distribution. The equation then became:

$$
f\left(x_{1}, x_{2}, x_{3}\right)=\text { Magnitude Factor } \times 0.1139\left[x_{1}+x_{2}+x_{3}\right]
$$

Table 4 summarizes the magnitude factors that were used to conduct further iterations of the linear function.

Table 4: Magnitude Factor Iteration Scheme of the Linear Function for the NSII flame

\begin{tabular}{|c|c|c|}
\hline \multirow{2}{*}{ Magnitude Factor } & \multicolumn{2}{|c|}{ Soot Volume Fraction (ppm) } \\
\cline { 2 - 3 } & Wings & Centerline \\
\hline 0.01 & 8.4497 & 3.108 \\
\hline 0.001 & 5.9686 & 2.6444 \\
\hline 0.0001 & 4.4386 & 2.3868 \\
\hline
\end{tabular}

Table 4 shows that a magnitude factor of 0.01 resulted in values close to the original model with the soot volume fraction along the wings having decreased while the centerline had increased. Further iterations were then required to increase the wing soot volume fraction prediction such that the simulation goal was met. Seeing as the difference between the predicted soot volume fractions of the original model and the results corresponding to a magnitude factor of 0.01 was of a relatively small order, the results were iterated further by smaller factors. It was decided that the range of values would be from 1.5 to 3.5 with increments of 0.5 and would be multiplied by the updated linear function.

The equation then became:

$$
f\left(x_{1}, x_{2}, x_{3}\right)=\text { Correction Factor } \times 0.01 \times 0.1139\left[x_{1}+x_{2}+x_{3}\right]
$$


Table 5 summarizes the correction factors that were used to conduct further iterations of the linear function.

Table 5: Correction Factor Iteration Scheme of the Linear Function for the NSII flame

\begin{tabular}{|c|c|c|}
\hline \multirow{2}{*}{ Correction Factor } & \multicolumn{2}{|c|}{ Soot Volume Fraction (ppm) } \\
\cline { 2 - 3 } & Wings & Centerline \\
\hline 1.5 & 9.0507 & 3.1749 \\
\hline 2.0 & 9.5093 & 3.2126 \\
\hline 2.5 & 9.9558 & 3.2210 \\
\hline 3.0 & 10.210 & 3.2320 \\
\hline 3.5 & 10.527 & 3.2765 \\
\hline
\end{tabular}

The results of these iterations yielded a value for $C_{1}$ wherein the peak soot volume fraction along the wings was close to the original model results while the centerline had increased. From Table 5, the correction factor that achieved this was 2.0, which made the collision efficiency equation become:

$$
\begin{gathered}
f\left(x_{1}, x_{2}, x_{3}\right)=2 \times 0.01 \times 0.1139\left[x_{1}+x_{2}+x_{3}\right] \\
f\left(x_{1}, x_{2}, x_{3}\right)=0.002278\left[x_{1}+x_{2}+x_{3}\right]
\end{gathered}
$$

Figure 18 shows the results of using the updated linear function to prescribe collision efficiency for the NSII flame plotted alongside the original model results and the experimental values. The soot volume fraction along the wings remains relatively close to the original model results. However, along the centerline, significant shifting of the peak can be observed. In contrast to the collision based aliphatic inception model best results, the shifting occurred along the wings whereas now the shifting is occurring along the centerline. 


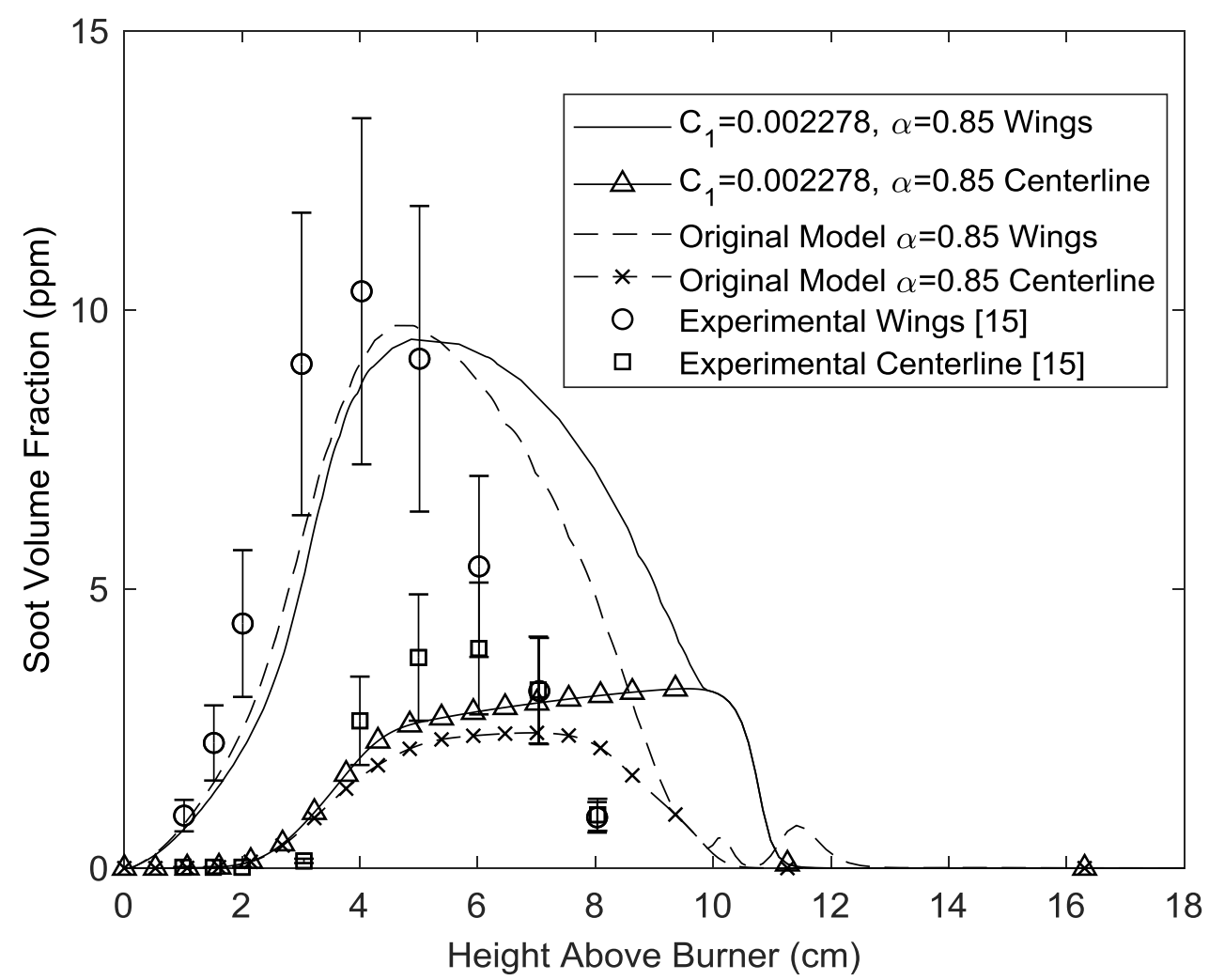

Figure 18: Predicted soot volume fraction as a function of height above the burner for the model with a collision efficiency prescribed by $f(x 1, x 2, x 3)=0.002278[x 1+x 2+x 3]$ along the wings and centerline of the NSII flame. The results of the original model without aliphatic inception are shown alongside experimental results from [15]. The surface reactivity was constant between the two models and had a value of 0.85 . The collision efficiency for the original model was a constant value of $1 \mathrm{e}-4$.

The last step was to find a $C_{1}$ that would achieve the desired results for a surface reactivity of 0.3 . This was desired in order to be able to compare the results of the stabilization model with the inception model results. Table 6 summarizes the iterations that were conducted to achieve the simulation goal outlined in the overview with a surface reactivity of 0.3 . Figure 19 shows the results of using $f(x)=2.6 * 10 * 0.002278[x]$ to prescribe the collision efficiency with a surface reactivity of 0.3 for the NSII flame plotted alongside the original model results and the experimental values. When comparing the results for the two computed functions seen in Figures 18 and 19, the surface reactivity appears to not have affected the soot distributions along both the wings and centerline. The peak wings were kept the same as the surface reactivity was 
lowered, but the collision efficiency was increased to compensate for less surface growth. This same effect may have been visible in the inception model had 2 solutions been generated for different surface reactivities. In principle this would make sense as both of the models are contributing to inception and not to surface growth.

Table 6: Iteration Scheme of the Linear Function for NSII Flame with an $\alpha=0.3$

\begin{tabular}{|c|c|c|}
\hline \multirow{2}{*}{ Equation } & \multicolumn{2}{|c|}{ Soot Volume Fraction (ppm) } \\
\cline { 2 - 3 } & Wings & Centerline \\
\hline$f(x)=0.002278[x]$ & 5.9260 & 2.6469 \\
\hline$f(x)=10 * 0.002278[x]$ & 8.2709 & 3.1007 \\
\hline$f(x)=1.8 * 10 * 0.002278[x]$ & 9.1109 & 3.1726 \\
\hline$f(x)=2.0 * 10 * 0.002278[x]$ & 9.2460 & 3.1429 \\
\hline$f(x)=2.2 * 10 * 0.002278[x]$ & 9.4180 & 3.1920 \\
\hline$f(x)=2.4 * 10 * 0.002278[x]$ & 9.5664 & 3.1984 \\
\hline$f(x)=2.6 * 10 * 0.002278[x]$ & 9.7057 & 3.2051 \\
\hline
\end{tabular}

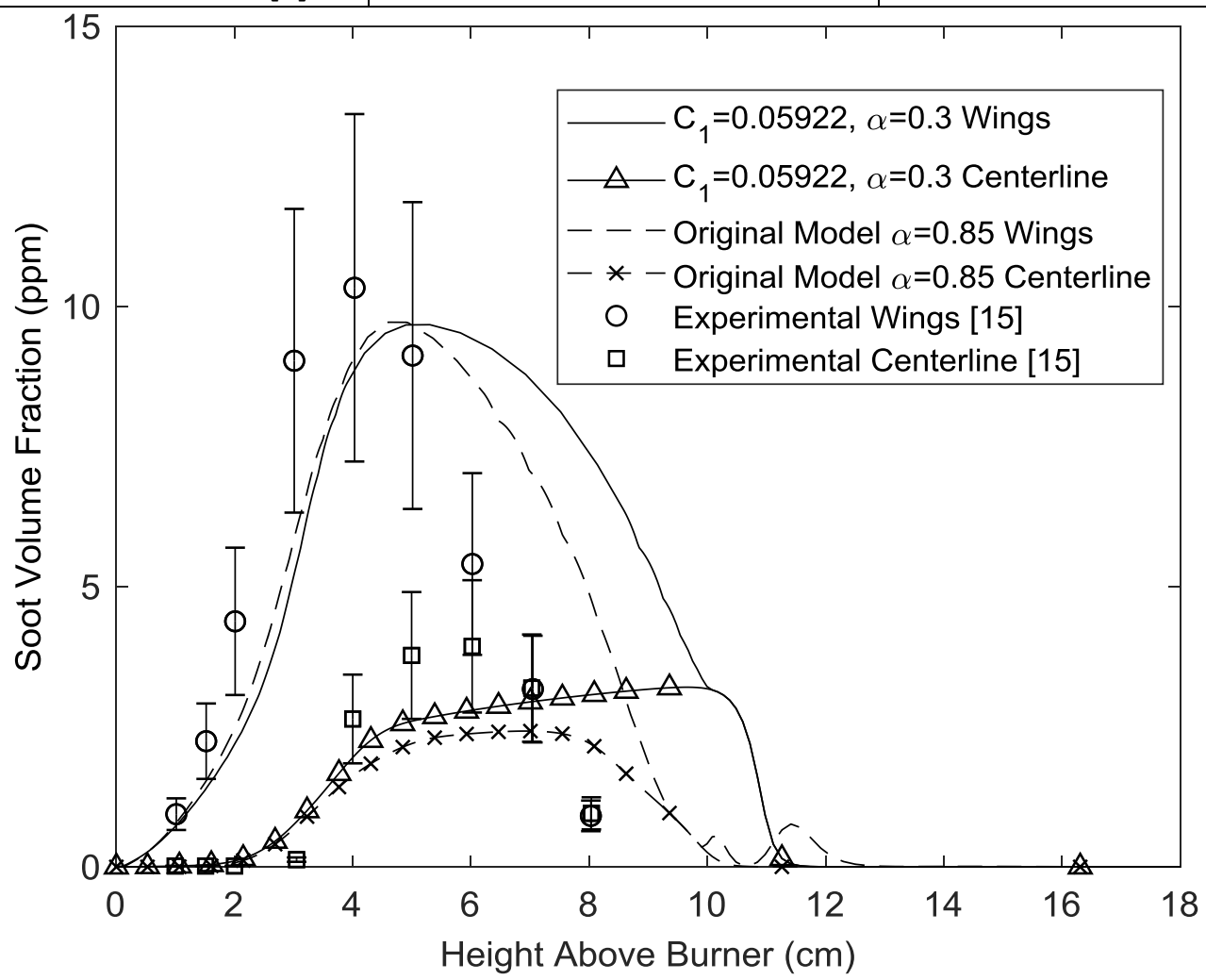

Figure 19: Predicted soot volume fraction as a function of height above the burner for the model with a collision efficiency prescribed by $f(x 1, x 2, x 3)=0.059228[x 1+x 2+x 3]$ along the wings and centerline of the NSII flame. The results of the original model without aliphatic inception are shown alongside experimental results from [15]. The surface reactivity for the original model was 0.85 whereas the stabilization model used a surface reactivity of 0.3 .

The collision efficiency for the original model was a constant value of $1 \mathrm{e}-4$. 


\subsection{Model Comparison with Experimental Results}

The two models that were generated for this body of work differed in the ways that the aliphatics were moved from the gaseous phase to the solid phase. One model assumed that aliphatic collisions resulted in the inception of soot and was implemented alongside PAH based inception. The other model assumed that aliphatics directly influenced PAH based inception by stabilizing the collisions between large PAHs. Figure 20 shows the best results of the simulations that were carried out for both of the models plotted alongside the original model and the experimental results for the NSII flame. Both models were capable of predicting the peak soot volume fraction along the wings, however, only the stabilization model was able to maintain the location accuracy. The inception model had a shifted peak. What is interesting to note is that at about $9 \mathrm{~cm}$ above the burner the pathline of maximum soot coincides with the centerline. Figure 21 is almost identical to Figure 20 but is plotted along the centerline instead of the wings. Both models were capable of improving the centerline prediction while maintaining accuracy along the wings. However, the shifting of the peak soot volume fraction had changed between the models as the inception model shared the same peak location as the original model. The stabilization model predicted a higher peak soot volume fraction; however, the peak had shifted. Lastly, the oxidation of soot was predicted to occur further downstream by both models. In conclusion, both models were capable of achieving the simulation goals with each having a unique impact on the soot distributions in different regions of the flame. 


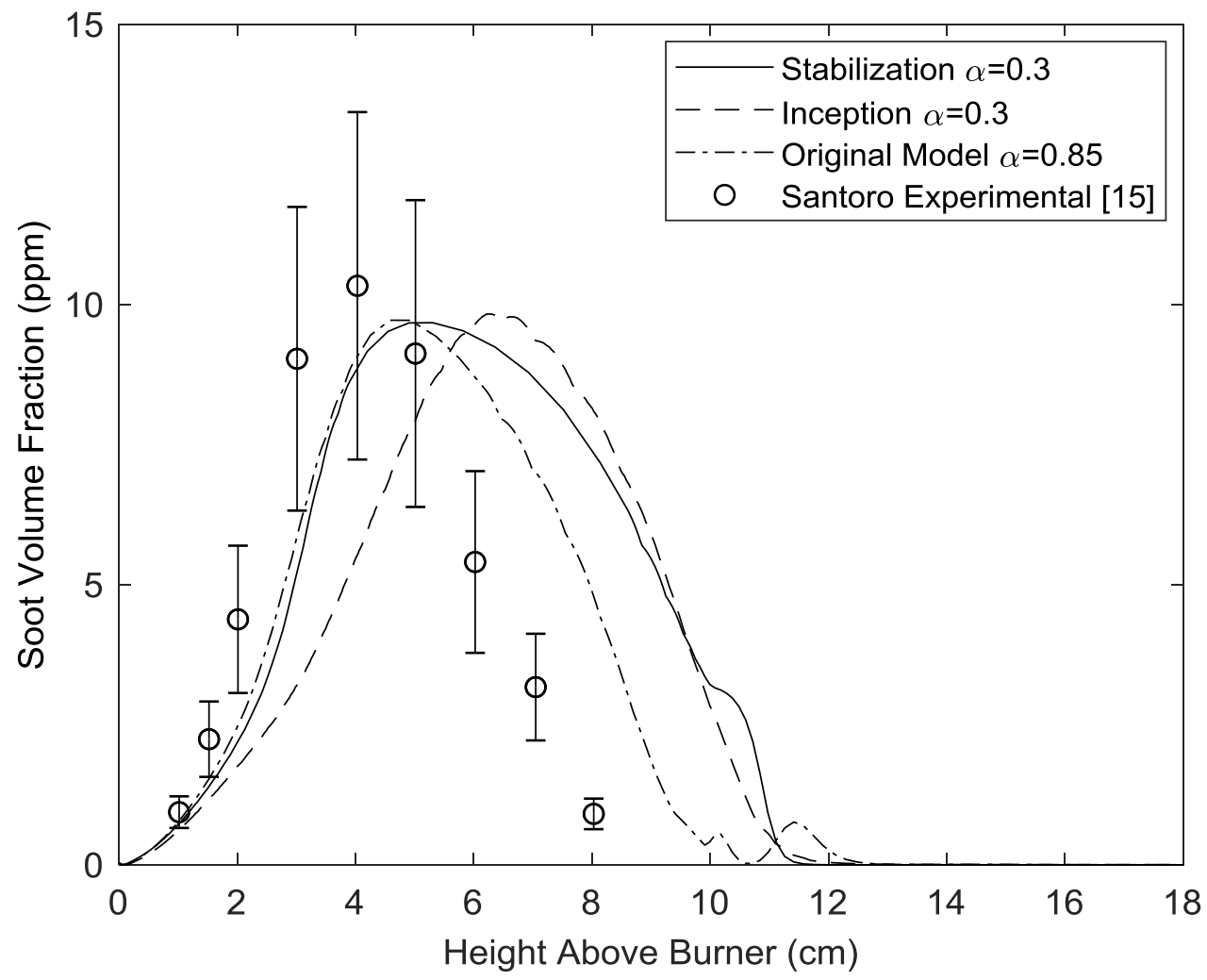

Figure 20: Predicted soot volume fraction as a function of height above the burner for the best results of the stabilization model, inception model, original model, and experimental results from [15]. 


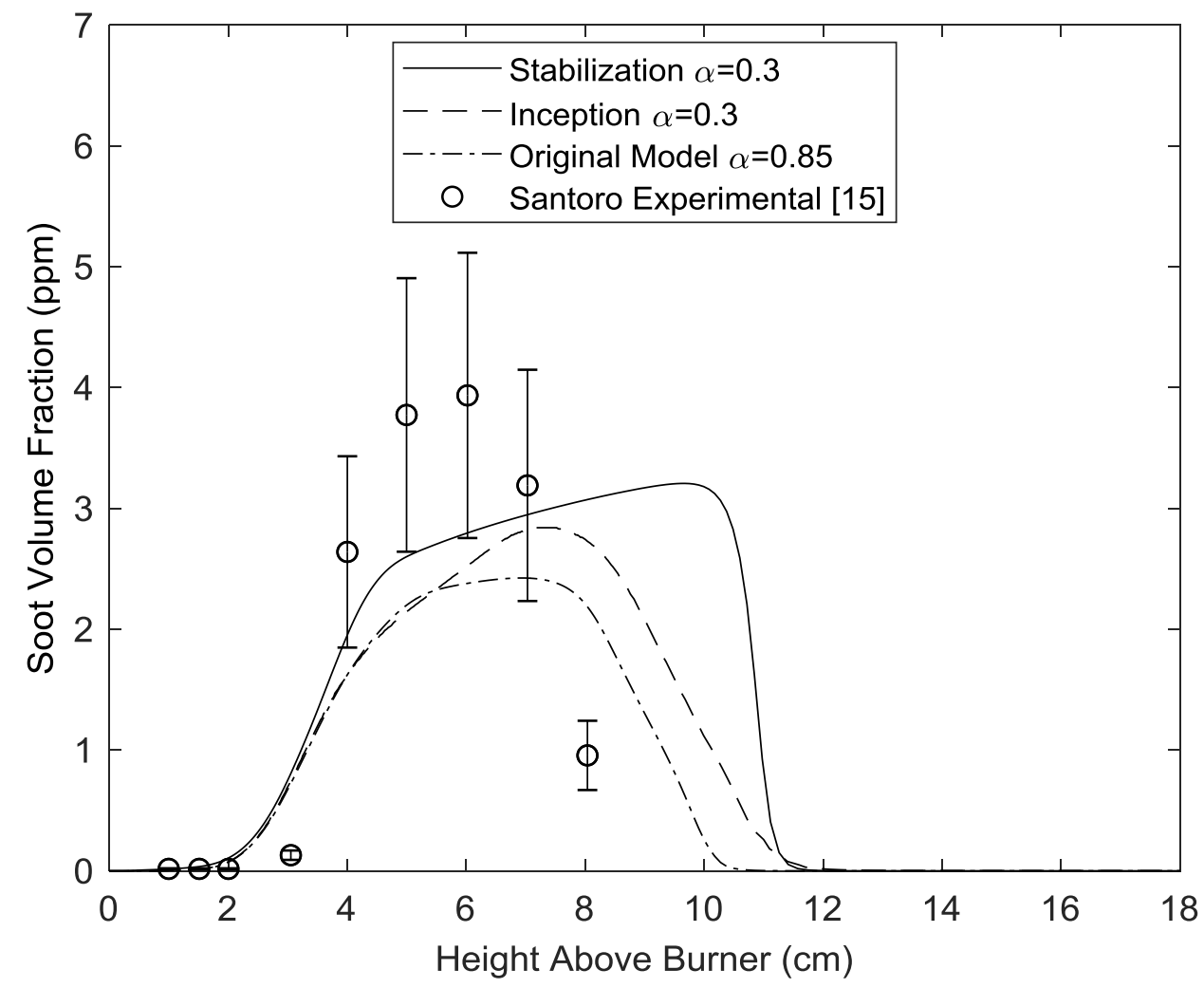

Figure 21: Predicted soot volume fraction as a function of height above the burner for the best results of the stabilization model, inception model, original model, and experimental results from [15]. 


\section{Chapter 5 - Closure}

\subsection{Conclusions}

The motivation behind this dissertation was to address the tendency of numerical models to underpredict the soot volume fraction along the centerline of coflow laminar diffusion flames. Recent literature suggested that nascent soot can be rich in aliphatics and that soot mass can grow without the presence of gas phase hydrogen atoms [16]. Furthermore, soot precursor species with aliphatic chains containing 3 carbons and higher were more likely to result in dimerization than when compared to the same species without these aliphatic chains $[20,21]$. Two novel inception models have been developed and tested on various laminar diffusion flames. The purpose of those models being to assess the influence that aliphatic based inception models have on soot formation to potentially address the underprediction of the soot distribution along the centerline of laminar diffusion flames.

Collision based aliphatic inception was developed using an existing framework for larger PAHs but applied to aliphatics. The aliphatic inception mechanism was combined with PAH inception and implemented in the CoFlame code. The influence that a collision based aliphatic inception model would have on the soot volume fraction distribution for coflow ethylene/air and methane/air laminar diffusion flames was investigated. It was found that for the ethylene flame, a surface reactivity, $\alpha$ of 0.3 and a collision efficiency, $\beta$ of $1 \mathrm{e}-11$ resulted in an increase in the peak soot volume fraction along the centerline, better predicting experimental values, while the predicted peak soot volume fraction along the wings was not degraded. For the methane flame, using the same $\beta$ of $1 \mathrm{e}-11$ resulted in an increase in both the centerline and wings of the flame as compared to using the same $\alpha$ in a soot model without collision based aliphatic inception. 
Collision based aliphatic stabilization differed from the aliphatic inception model in the manner in which the stabilization model transferred mass from the gas phase into the solid phase. Instead of two aliphatics colliding and forming soot, the stabilization model proposed that aliphatics facilitated the collisions between large PAHs. Through this, when an successful collision between two large PAHs resulted in the formation of soot, the aliphatic species of 3 carbons and higher were simultaneously moved to the solid phase along with these PAHs. Investigation was carried out to assess the effects that a functional form of collision efficiency for PAHs would have on the soot volume fraction distributions for the ethylene/air flame. It was determined that a linear function did exist wherein improvement could be seen along the centerline soot volume fraction distribution without degrading the peak prediction along the wings.

\subsection{Future Work}

The developed novel models have acted as a proof of concept in that aliphatics are able to influence the centerline soot volume fraction while maintaining accuracy along the wings for the ethylene flames. In the current state, the models that were generated are not of a fully fundamental nature that is derived from first principles as soot formation is a complex process. Therefore, the models have significant areas that they can be improved upon and some recommendations would include:

1. Testing the applicability of these models to other combustion systems, in particular those for which model predictions do not completely explain or characterize experimental data. To test the validity of these models further, the results should be expanded to other flame systems in order to ensure applicability to a wide variety of flames and burner configurations. For example, the flames studied by Smooke et al. [61], which are coflow ethylene/air/nitrogen 
laminar diffusion flames with a variety of nitrogen dilution ratios and a different burner configuration. In particular, the most heavily diluted flames in [61] demonstrate strong soot formation along the centerline that has not been well captured by CoFlame to date. Furthermore, the results of these models should be applied to the other pure ethylene/air diffusion flames studied by Santoro et al. [15] to observe the effects these models would have with varying fuel and oxidizer velocities. The other ethylene flames suffer from the same predictive discrepancies as the NSII flame.

2. Deriving the Arrhenius kinetic rates for the stabilization model, based on molecular dynamics simulations, and implementing them into CoFlame. This would improve the stabilization model significantly by having derived values for the reaction rates rather than having iteratively obtained solutions. Furthermore, it would be more physically justifiable if an activation energy barrier for these reactions was present and the chemical reaction was reversible.

3. Tracking the portion of aliphatics that are moved from the gas phase to the soot solid phase and correlating those results with what is seen experimentally in transmission electron microscopy. This would allow for comparisons to be drawn for the reaction rates of the aliphatics between the numerical model and experimental results.

4. A potential pathway of interest may also be to consider aliphatic condensation and determine if it plays a significant role in soot formation. The condensation model would in essence function similar to PAH condensation wherein a portion of the collisions between soot particles and aliphatics would result in sticking. This model would have a fundamentally different nature when compared to the models that were generated for this body of work. 
The difference being in that the interactions of the aliphatics with soot would be between the gas and solid phase. The models that were generated for this work operated only in the gas phase. Adding these aliphatics to surface growth may influence the centerline if those added aliphatics reside in that region of the flame. 


\section{Bibliography}

[1] N. Janssen, M. Gerlofs-Nijland, T. Lanki, R. Salonen, F. Cassee, G. Hoek, P. Fischer, B. Brunekreef and M. Krzyzanowski, Health Effects of Black Carbon, World Health Organization, 2012.

[2] N. Janssen, G. Hoek, M. Simic-Lawson, P. Fischer, L. V. Bree, H. Brink, M. Keuken, R. Atkinson, H. Anderson, B. Brunekreef and F. Cassee, "Black Carbon as an Additional Indicator of the Adverse Health Effects of Airborne Particles Compared with PM10 and PM2.5," Environmental Health Perspectives, vol. 119, no. 12, pp. 1691-1699, 2012.

[3] S. Vedal, "Ambient Particles and Health: Lines that Divide," Journal of the Air and Waste Management Association, vol. 47, no. 5, pp. 551-581, 1997.

[4] J. Hansen and L. Nazarenko, "Soot Climate Forcing via Snow and Ice Albedos," Proceedings of the National Academy of Sciences of the United States of America, vol. 101, no. 2, pp. 423-428, 2004.

[5] J. Penga, M. Hua, S. Guoa, Z. Dua, J. Zhenga, D.Shanga, M. Zamorab, L. Zenga, M. Shaob, Y. Wua, J. Zhengb, Y. Wangc, C. Glenb, D. Collinsb, M. Molinad and R. Zhanga, "Markedly enhanced absorption and direct radiative forcing of black carbon under polluted urban environments," Proceedings of the National Academy of Sciences, vol. 113, no. 16, p. 4266, 2016.

[6] B. Haynes and G. Wagner, "Soot Formation," Progress in Energy and Combustion Science, vol. 7, no. 4, pp. 229-273, 1981.

[7] M. Fairweather, W. Jones, H. Ledin and R. Lindstedt, "Prediction of Soot Formation in Turbulent Non-Premixed Propane Flames," Proceedings of the Combustion Institute, vol. 24, no. 1, pp. 1067-1074, 1992.

[8] I. M. Kennedy, "Models of Soot Formation and Oxidation," Progress in Energy and Combustion Science, vol. 23, no. 2, pp. 95-132, 1997.

[9] M. Frenklach and H. Wang, "Detailed Modelling of Soot Particle Nucleation and Growth," Symposium (International) on Combustion, vol. 23, no. 1, pp. 1559-1566, 1991.

[10] V. Chernov, Q. Zhang, M. Thomson and S. Dworkin, "Numerical Investigation of Soot Formation Mechanism in Partially-Premixed Ethylene-Air Co-Flow Flames," Combustion and Flame, vol. 159, no. 9, pp. 2789-2798, 2012. 
[11] S. Dworkin, Q. Zhang, M. Thomson, N. Slavinskaya and U. Riedel, "Application of an Enhanced PAH Growth Model to Soot Formation in Laminar Coflow Ethylene/air Diffusion Flame," Combustion and Flame, vol. 158, no. 9, pp. 1682-1695, 2011.

[12] V. Chernov, M. Thomson, S. Dworkin, N. Slavinskaya and U. Riedel, "Soot Formation with C1 and C2 Fuels using an Improved Chemical Mechanism for PAH Growth," Combustion and Flame, vol. 161, no. 2, p. 592, 2014.

[13] J. Appel, H. Bockhorn and M. Frenklach, "Kinetic Modeling of Soot Formation with Detailed Chemistry and Physics: Laminar Premixed Flames of C2 Hydrocarbons," Combustion and Flame, vol. 121, no. 1, pp. 122-136, 2000.

[14] N. Marinov, W. Pitz, C. Westbrooke, A. Vincitore, M. Castaldi and S. Senkan, "Aromatic and Polycyclic Aromatic Hydrocarbon Formation in a Laminar Premixed n-Butane Flame.," Combustion and Flame, vol. 114, no. 1, pp. 192-213, 1998.

[15] R. Santoro, T. Yeh, J. Horvath and H. Semerjian, "The Transport and Growth of Soot Particles in Laminar Diffusion Flames," Combustion Science and Technology, vol. 53, no. 1, pp. 89-115, 1987.

[16] H. Wang, "Formation of Nascent Soot and Other Condensed-Phase Materials in Flames," Proceedings of the Combustion Institute, vol. 33, no. 1, pp. 41-67, 2011.

[17] H. Wang, B. Zhao, B. Wyslouzil and K. Streletzky, "Small-angle neutron scattering of soot formed in laminar premixed ethylene flames," Proceedings of the Combustion Institute, vol. 29, no. 1, pp. 2749-2757, 2002.

[18] B. Zhao, K. Uchikawa and H. Wang, "Proceedings of the Combustion Institute," A Comparative Study of Nanoparticles in Premixed Flames by Scanning Mobility Particle Sizer, Small Angle Neutron Scattering, and Transmission Electron Microscopy, vol. 31, no. 1, pp. 851-860, 2007.

[19] B. Öktem, M. Tolocka, B. Zhao, H. Wang and M. Johnston, "Chemical species associated with the early stage of soot growth in a laminar premixed ethylene-oxygen-argon flame," Combustion and Flame, vol. 142, no. 4, pp. 364-373, 2005.

[20] P. Elvati and V. Angela, "Proceedings of the Combustion Institute," Thermodynamics of Poly-Aromatic Hydrocarbon Clustering and the Effects of Substituted Aliphatic Chains, vol. 34, no. 1, pp. 1837-1843, 2013.

[21] S. Chung and V. Angela, "Peri-Condensed Aromatics with Aliphatic Chains as Key Intermediates for the Nucleation of Aromatic Hydrocarbons," Proceedings of the Combustion Institute, vol. 33, no. 1, pp. 693-700, 2011. 
[22] V. Angela, "Modeling of Soot Particle Inception in Aromatic and Aliphatic Premixed Flames," Combustion and Flame, vol. 139, no. 1, pp. 279-287, 2004.

[23] N. Eaves, "The Effect of Reversibility and High Pressure on Soot Formation," PhD Thesis, 2016.

[24] R. J. Santoro, H. G. Semerjian and R. A. Dobbins, "Soot particle measurements in diffusion flames," Combustion and Flame, vol. 51, no. 1, pp. 203-218, 1983.

[25] N. A. Eaves, A. Veshkini, C. Riese, Q. Zhanga, S. B. Dworkin and M. J. Thomson, "A numerical study of high pressure, laminar, sooting, ethane-air coflow diffusion flames," Combustion and Flame, vol. 159, no. 10, pp. 3179-3190, 2012.

[26] F. Liu, H. Guo, G. Smallwood and Ö. Gülder, "Numerical Modelling of Soot Formation and Oxidation in Laminar Coflow Non-Smoking and Smoking Ethylene Diffusion Flames," Combustion Theory and Modelling, vol. 7, no. 1, pp. 301-315, 2003.

[27] C. Megaridis and R. Dobbins, "Soot Aerosol Dynamics in a Laminar Ethylene Diffusion Flame," Proceedings of the Combustion Institute, vol. 22, no. 1, pp. 353-362, 1988.

[28] C. Megaridis and R. Dobbins, " Comparison of Soot Growth and Oxidation in Smoking and Non-Smoking Ethylene Diffusion Flames," Combustion Science Technology, vol. 66, no. 1, pp. 1-16, 1989.

[29] K.-O. Lee, C. M. Megaridis, S. Zelepouga, A. V. Saveliev, L. A.Kennedy, O. Charon and F. Ammouri, "Soot formation effects of oxygen concentration in the oxidizer stream of laminar coannular nonpremixed methane/air flames," Combustion and Flame, vol. 121, no. 1, pp. 323-333, 2000.

[30] F. Liu, G. J. Smallwood and O. L. Gulder, "Band lumping strategy for radiation heat transfer calculations using a narrowband model," Journal of Thermophysics and Heat Transfer, vol. 2, no. 278-281, p. 14, 2000.

[31] K. H. Lee and R. Viskanta, "Prediction of spectral radiative transfer in a condensed cylindrical medium using discrete ordinates method," Journal of Quantitative Spectroscopy and Radiative Transfer, vol. 58, no. 3, pp. 329-345, 1997.

[32] C. P. Thurgood, A. Pollard and H. A. Becker, "Tn quadrature set for the discrete ordinates method.," Journal of Heat Transfer, vol. 4, no. 1068-1070, p. 117, 1995.

[33] N. Eaves, "The Effect of Pressure and Conjugate Heat Transfer on Soot," MASc Thesis, 2012. 
[34] "The International Sooting Flame Workshop," https://www.adelaide.edu.au/cet/isfworkshop/.

[35] S. V. Patankar, "Numerical Heat Transfer and Fluid Flow," Hemisphere, 1980.

[36] R. Kee, J. Miller and T. Jefferson, "Chemkin: A general purpose, problem independent, transportable, fortran chemical kinetics code package," Technical Report SAN80-8003, 1980.

[37] R. Kee, F. Rupley and J. Miller, "A fortran chemical kinetics package for the analysis of gasphase chemical kinetics," Technical Report SAN89-8009, 1989.

[38] Q. Zhang, "Detailed Modeling of Soot Formation/Oxidation in Laminar Coflow Diffusion Flames," PhD Thesis, University of Toronto, Toronto, Canada, 2009.

[39] M. Frenklach, D. W. Clary, W. C. G. Jr. and S. E. Stein, "Detailed kinetic modeling of soot formation in shock-tube pyrolysis of acetylene," Proceedings of the Combustion Institute, vol. 20, no. 1, pp. 887-901, 1984.

[40] C. F. Melius and J. A. Miller, "Kinetic and thermodynamic issues in the formation of aromatic compounds in flames of aliphatic fuels," Combustion and Flame, vol. 91, no. 1, pp. 21-39, 1992.

[41] S. E. Stein, J. A. Walker, M. M. Suryan and A. Fahr, "A new path to benzene in flames," Proceedings of the Combustion Institute, vol. 23, no. 1, pp. 85-90, 1991.

[42] C. F. Melius, M. E. Colvin, N. M. Marinov, W. Pitz and S. M. Senkan, "Reaction mechanisms in aromatic hydrocarbon formation involving the c5h5 cyclopentadienyl moiety," Proceedings of the Combustion Institute, vol. 1, no. 1, pp. 685-692, 1996.

[43] N. A. Slavinskaya and P. Frank, "A modelling study of aromatic soot precursors formation in laminar methane and ethene flames.," Combustion and Flame, vol. 156, no. 9, pp. 17051722, 2009.

[44] K. Homann, "Fullerenes and soot formation - new pathways to large particles in flames," Angewandte Chemie - International Edition, vol. 37, no. 18, pp. 2434-2451, 1998.

[45] A. D'Anna, "Combustion-formed nanoparticles," Proceedings of the Combustion Institute, vol. 32, no. 1, pp. 593-613, 2009.

[46] M. Saffaripour, A. Veshkini, M. Kholghy and M. Thomson., "Experimental Investigation and Detailed Modeling of Soot Aggregate Formation and Size Distribution in Laminar Coflow 
Diffusion Flames of Jet A-1, a Synthetic Kerosene, and n-decane," Combustion and Flame, vol. 161, no. 3, pp. 848-863, 2014.

[47] S. H. Park, S. N. Rogak, W. K. Bushe, J. Z. Wen and M. J. Thomson, "An aerosol model to predict size and structure of soot particles," Combustion Theory and Modelling, vol. 9, no. 3, pp. 499-513, 2005.

[48] J. Z. Wen, M. J. Thomson, M. F. Lightstone, S. H. Park and S. N. Rogak, "An improved moving sectional aerosol model of soot formation in a plug flow reactor," Combustion Science and Technology, vol. 178, no. 5, pp. 921-951, 2006.

[49] M. Zurita-Gotor and D. E. Rosner, "Effective diameters for collisions of fractal-like aggregates: Recommendations for improved aerosol coagulation frequency predictions," Journal of colloid and interface science, vol. 255, no. 1, pp. 10-26, 2002.

[50] C. M. Sorensen and G. M. Wang, "Note on the correction for diffusion and drag in the slip regime," Aerosol Science and Technology, vol. 33, no. 4, pp. 353-356, 2000.

[51] K. Naumann, "Cosima - a computer program simulating the dynamics of fractal aerosols," Journal of Aerosol Science, vol. 34, no. 10, pp. 1371-1397, 2003.

[52] A. Khosousi and S. B. Dworkin, "Soot surface reactivity during surface growth and oxidation in laminar diffusion flames," Combustion and Flame, vol. 162, no. 12, pp. 45234532, 2015.

[53] A. Veshkini, S. Dworkin and M. Thomson., "Understanding Soot Particle Size Evolution in Laminar Ethylene/Air Diffusion Flames Using Novel Soot Coalescence Models," Combustion Theory and Modelling, vol. 20, no. 4, pp. 707-734, 2016.

[54] A. Khosousi and S. B. Dworkin, "Detailed modelling of soot oxidation by $\mathrm{O} 2$ and $\mathrm{OH}$ in laminar diffusion flames," Proceedings of the Combustion Institute, vol. 35, no. 2, pp. 1903-1910, 2015.

[55] K. G. Neoh and J. B. Howard, "Particulate Carbon-Formation during Combustion," Plenum, New York, pp. 77-261, 1981.

[56] Q. Zhang, M. Thomson, H. G. Liu and G. Smallwood, "Modeling of Oxidation-Driven Soot Aggregate Fragmentation in a Laminar Coflow Diffusion Flame," Combustion Science and Technology, vol. 182, no. 4-6, pp. 491-504, 2010.

[57] C. P. Arana, M. Pontoni, S. Sen and I. K. Puri, "Field measurements of soot volume fractions in laminar partially premixed coflow ethylene/air flames," Combustion and Flame, vol. 138, no. 4, pp. 362-372, 2004. 
[58] C. S. McEnally and L. D. Pfefferle, "Experimental study of nonfuel hydrocarbons and soot in coflowing partially premixed ethylene/air flames," Combustion and Flame, vol. 121, no. 4, pp. 575-592, 2000.

[59] Ü. Köylü, C. McEnally, D. Rosner and L. Pfefferle, "Simultaneous measurements of soot volume fraction and particle size / microstructure in flames using a thermophoretic sampling technique," Combustion and Flame, vol. 110, no. 4, pp. 494-507, 1997.

[60] A. Veshkini, S. B. Dworkin and M. J. Thomson, "A soot particle surface reactivity model applied to a wide range of laminar ethylene/air flames," Combustion and Flame, vol. 161, no. 12, pp. 3191-3200, 2014.

[61] M. Smooke, M. Long, B. Connelly, M. Colket and R. Hall, "Soot Formation in Laminar Diffusion Flames," Combustion and Flame, vol. 143, no. 1, pp. 613-628, 2005. 